Supporting Information

\title{
Design and Synthesis of a Potent Phorboxazole C(11-15) Acetal Analogue
}

\author{
Amos B. Smith, III, ${ }^{* \dagger}$ Thomas M. Razler, ${ }^{\dagger}$ Regina M. Meis, ${ }^{\dagger}$ and George R. Pettit ${ }^{\ddagger}$
}

${ }^{\dagger}$ Department of Chemistry, Monell Chemical Senses Center, and Laboratory for Research on the Structure of Matter, University of Pennsylvania, Philadelphia, Pennsylvania 19104, U.S.A.

${ }^{\sharp}$ Cancer Research Institute, Arizona State University, Tempe, Arizona 85287 
Material and Methods: All solvents were reagent grade. Diethyl ether $\left(\mathrm{Et}_{2} \mathrm{O}\right)$ and tetrahydrofuran (THF) were freshly distilled from sodium/benzophenone under argon. $n$ Butyllithium and $t$-butyllithium were purchased from Aldrich. Reactions were magnetically stirred and monitored by thin layer chromatography (TLC) with $0.25 \mathrm{~mm} \mathrm{E}$. Merck pre-coated silica gel plates. Flash chromatography was performed with silica gel 60 (particle size $0.040-0.062 \mathrm{~mm}$ ) supplied by Silicycle and Sorbent Technologies. Yields refer to chromatographically and spectroscopically pure compounds, unless otherwise stated. Infrared spectra were recorded on a Jasco Model FT/IR-480 Plus spectrometer. Proton and carbon-13 NMR spectra were recorded on a Bruker AMX-500 spectrometer. Chemical shifts are reported relative to either chloroform ( $\delta 7.26)$ or benzene $(\delta 7.16)$ for ${ }^{1} \mathrm{HNMR}$ and either chloroform $(\delta 77.0)$ or benzene $(\delta 128.0)$ for ${ }^{13}$ CNMR. Optical rotations were measured on a Perkin-Elmer model 241 polarimeter. High resolution mass spectra were measured at the University of Pennsylvania Mass Spectrometry Service Center.

\section{Experimental Procedures}

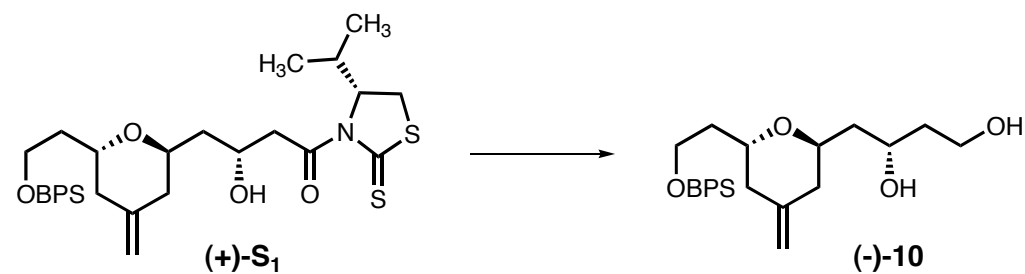

Diol (-)-10: To a solution of $\beta$-Hydroxyimide $(+)-S_{1}(1.0 \mathrm{~g}, 1.56 \mathrm{mmol})$ in diethyl ether (6.25 mL) at $0{ }^{\circ} \mathrm{C}$ under argon was added $\mathrm{H}_{2} \mathrm{O}(0.031 \mathrm{~mL}, 1.72 \mathrm{mmol})$ and $\mathrm{LiBH}_{4}(0.037 \mathrm{~g}, 1.72$ $\mathrm{mmol}$ ) in one portion. After stirring at $0{ }^{\circ} \mathrm{C}$ for 10 minutes, the reaction was warmed to room temperature, stirred for 10 minutes, cooled back to $0{ }^{\circ} \mathrm{C}$ and quenched with $\mathrm{pH} 7$ phosphate buffer $(7 \mathrm{~mL})$. The layers of the biphasic mixture were separated and the aqueous layer was extracted with diethyl ether $(20 \mathrm{~mL}, 3 x)$. The combined organic extracts were washed with 
saturated aqueous sodium chloride $(20 \mathrm{~mL}, 1 \mathrm{x})$, dried over $\mathrm{MgSO}_{4}$, filtered and concentrated under reduced pressure. Purification via silica gel chromatography (50\% EtOAc/hexanes) afforded (-)-10 (0.604 g, 83\% yield) as a colorless oil: $[\alpha]_{\mathrm{D}}^{20}-18.3\left(c\right.$ 1.0, $\left.\mathrm{CHCl}_{3}\right)$; IR (neat) 3397 (b), 3070 (w), 2939 (s), 2857 (s), 1654 (w), 1472 (w), 1428 (s), 1389 (w), 1110 (s), 890 (m), 823 (m), $740(\mathrm{~m}), 701$ (s) $\mathrm{cm}^{-1} ;{ }^{1} \mathrm{HNMR}\left(500 \mathrm{MHz}, \mathrm{CDCl}_{3}\right) \delta 7.67(\mathrm{~m}, 4 \mathrm{H}), 7.40(\mathrm{~m}, 6 \mathrm{H}), 4.78(\mathrm{~s}, 1 \mathrm{H})$, $4.72(\mathrm{~s}, 1 \mathrm{H}), 4.25(\mathrm{~m}, 1 \mathrm{H}), 3.92(\mathrm{~m}, 1 \mathrm{H}), 3.89(\mathrm{~s}, 1 \mathrm{H}), 3.76(\mathrm{~m}, 4 \mathrm{H}), 3.67(\mathrm{ddd}, J=10.8,6.7,5.6$ $\mathrm{Hz}, 1 \mathrm{H}), 2.87$ (dd, $J=6.7,4.5 \mathrm{~Hz}, 1 \mathrm{H}), 2.45(\mathrm{dd}, J=13.4,5.6 \mathrm{~Hz}, 1 \mathrm{H}), 2.21(\mathrm{dd}, J=13.1,3.0 \mathrm{~Hz}$, 1H), $2.01(\mathrm{~m}, 2 \mathrm{H}), 1.94$ (dddd, $J=14.1,8.6,5.6,5.6 \mathrm{~Hz}, 1 \mathrm{H}), 1.75$ (ddd, $J=14.5,10.1,10.1 \mathrm{~Hz}$, 1H), $1.65(\mathrm{~m}, 3 \mathrm{H}), 1.43$ (ddd, $J=14.5,2.2,2.2 \mathrm{~Hz}, 1 \mathrm{H}), 1.05(\mathrm{~s}, 9 \mathrm{H}) ;{ }^{13} \mathrm{CNMR}\left(125 \mathrm{MHz}, \mathrm{CDCl}_{3}\right)$ $\delta 141.0,135.4,135.3,133.6,133.4,129.5,129.4,127.58,127.52,110.5,71.7,71.5,70.1,61.0$, $60.3,41.4,40.5,38.55,38.53,34.3,26.7,18.9$; high resolution mass spectrum $\left(\mathrm{ES}^{+}\right) \mathrm{m} / \mathrm{z}$ 491.2686 [(M+Na $)^{+}$; calcd for $\mathrm{C}_{28} \mathrm{H}_{40} \mathrm{O}_{4} \mathrm{SiNa:}$ 491.2593].

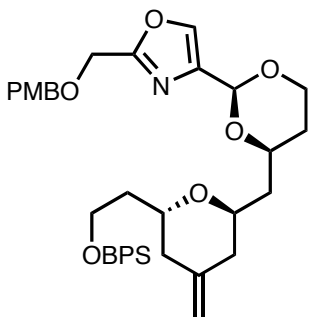

$(-)-12$

C(11-15) Acetal (-)-12: Under an argon atmosphere at $-78{ }^{\circ} \mathrm{C}, 1,1,1,3,3,3-$ hexamethydisilazane (HMDS) $(0.93 \mathrm{~mL}, 4.41 \mathrm{mmol})$ was added dropwise via syringe to a stirred solution of diol (-)-10 (1.97 g, $4.21 \mathrm{mmol})$ in $\mathrm{CH}_{2} \mathrm{Cl}_{2}(10 \mathrm{~mL})$. Following the addition, the reaction was warmed to room temperature. After twenty hours, the reaction was concentrated under reduced pressure followed by drying under vacuum (to remove excess 1,1,1,3,3,3hexamethyldisilazane) to provide the corresponding bis-silylated diol, which was used without further purification. Under an argon atmosphere at $0{ }^{\circ} \mathrm{C}$, trimethylsilyl trifluoromethanesulfonate (TMSOTf) $(0.148 \mathrm{~mL}, 0.82 \mathrm{mmol})$ was added dropwise to a solution of oxazole 11 (2.00 g, 8.11 $\mathrm{mmol}$ ) and the bis-silylated diol in $\mathrm{CH}_{2} \mathrm{Cl}_{2}(21 \mathrm{~mL})$. After stirring for fifteen hours, the reaction was 
cooled to $-78{ }^{\circ} \mathrm{C}$, quenched with triethylamine $(2.35 \mathrm{~mL}, 16.8 \mathrm{mmol})$, and warmed to room temperature over one hour. The reaction was diluted with saturated aqueous sodium bicarbonate (20 mL) and extracted with $\mathrm{CH}_{2} \mathrm{Cl}_{2}(10 \mathrm{~mL}, 3 \mathrm{x})$. The combined organic extracts were dried over $\mathrm{MgSO}_{4}$, filtered and concentrated under reduced pressure. Purification via silica gel chromatography (10\% EtOAc/hexanes $\rightarrow$ 20\% EtOAc/hexanes) afforded C(11-15) acetal (-)-12 $\left(2.52 \mathrm{~g}, 86 \%\right.$ yield) as a light yellow oil: $[\alpha]_{\mathrm{D}}^{20}-16.1\left(c \mathrm{0.5}, \mathrm{CHCl}_{3}\right)$; IR (neat) $3150(\mathrm{w}), 3070(\mathrm{~m})$, 2930 (s), 2856 (s), $1889(w), 1821(w), 1727(w), 1653(m), 1612(s), 1586(m), 1513(s), 1464$ (m), $1428(\mathrm{~s}), 1375(\mathrm{~m}), 1302(\mathrm{~m}), 1249(\mathrm{~s}), 1175(\mathrm{~m}), 1111(\mathrm{~s}), 1036(\mathrm{~s}), 822(\mathrm{~s}), 741(\mathrm{~m}), 704$ (s) $614(\mathrm{~m}) \mathrm{cm}^{-1}$; ${ }^{1} \mathrm{HNMR}\left(500 \mathrm{MHz}, \mathrm{CDCl}_{3}\right) \delta 7.66(\mathrm{~m}, 5 \mathrm{H}), 7.39(\mathrm{~m}, 6 \mathrm{H}), 7.26(\mathrm{~m}, 2 \mathrm{H}), 6.87(\mathrm{~m}$, 2H), $5.44(\mathrm{~s}, 1 \mathrm{H}), 4.75(\mathrm{~s}, 2 \mathrm{H}), 4.55(\mathrm{~s}, 2 \mathrm{H}), 4.52(\mathrm{~s}, 2 \mathrm{H}), 4.14(\mathrm{dd}, J=11.5,4.8 \mathrm{~Hz}, 1 \mathrm{H}), 4.03(\mathrm{~m}$, 1H), $3.87(\mathrm{~m}, 2 \mathrm{H}), 3.80(\mathrm{~s}, 3 \mathrm{H}), 3.72(\mathrm{~m}, 3 \mathrm{H}), 2.34$ (ddd, $J=13.0,3.4,3.4 \mathrm{~Hz}, 2 \mathrm{H}), 2.10$ (ddd, $J=$ 14.1, 8.2, $5.6 \mathrm{~Hz}, 1 \mathrm{H}), 2.00(\mathrm{~m}, 2 \mathrm{H}), 1.85(\mathrm{~m}, 1 \mathrm{H}), 1.73(\mathrm{~m}, 1 \mathrm{H}), 1.67(\mathrm{~m}, 2 \mathrm{H}), 1.51(\mathrm{~m}, 1 \mathrm{H}), 1.05$ (s, 9H); ${ }^{13} \mathrm{CNMR}\left(125 \mathrm{MHz}, \mathrm{CDCl}_{3}\right) \delta 161.1,159.4,141.8,139.1,136.9,135.5,133.82,133.79$, $129.7,129.6,129.2,127.63,127.58,113.84,113.81,110.4,96.1,74.0,72.5,68.9,67.8,66.8$, $63.5,60.6,55.2,39.7,39.6,39.0,36.4,30.7,29.6,26.8,19.1$; high resolution mass spectrum $\left(\mathrm{ES}^{+}\right) \mathrm{m} / \mathrm{z} 698.3538\left[(\mathrm{M}+\mathrm{H})^{+}\right.$; calcd for $\left.\mathrm{C}_{41} \mathrm{H}_{51} \mathrm{NO}_{7} \mathrm{SiH}: 698.3513\right]$.

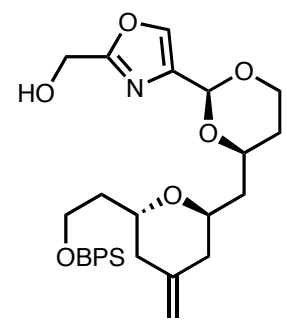

$(-)-\mathbf{S}_{2}$

Alcohol Tricycle (-)-S $\mathbf{S}_{2}$ : 2,3-Dichloro-5,6-dicyano-1,4-benzoquinone (DDQ) (1.24 g, 5.47 $\mathrm{mmol})$ was added as one portion to a solution of $\mathrm{C}(11-15)$ acetal $(-)-12(1.80 \mathrm{~g}, 2.60 \mathrm{mmol})$ in $\mathrm{CH}_{2} \mathrm{Cl}_{2}(260 \mathrm{~mL})$ and $\mathrm{H}_{2} \mathrm{O}(17.4 \mathrm{~mL})$ at room temperature under an argon atmosphere. After stirring for twelve hours, the reaction was quenched via dropwise addition of saturated aqueous sodium bicarbonate $(30 \mathrm{~mL})$. The layers of the biphasic mixture were separated and the aqueous 
layer was extracted with $\mathrm{CH}_{2} \mathrm{Cl}_{2}(20 \mathrm{~mL}, 4 \mathrm{x})$. The combined organic extracts were dried over $\mathrm{MgSO}_{4}$, filtered and concentrated under reduced pressure. Purification via silica gel chromatography (50\% EtOAc/hexanes) afforded alcohol tricycle (-)-S $\mathbf{S}_{2}(1.26 \mathrm{~g}, 84 \%$ yield) as a light yellow oil: $[\alpha]_{\mathrm{D}}^{20}-18.9\left(c\right.$ 0.5, $\left.\mathrm{CHCl}_{3}\right)$; IR (neat) $3376(\mathrm{~b}), 3158(\mathrm{w}), 3071(\mathrm{~m}), 2931(\mathrm{~s}), 2857$ (s), $1901(w), 1828(w), 1652(m), 1578(m), 1471(m), 1428(s), 1375(m), 1237(m), 1185(m)$, 1112 (s), 1038 (s), 1010 (m), 937 (w), 893 (w), 823 (s), 757 (s), 704 (s), 614 (m) cm ${ }^{-1} ;{ }^{1} \mathrm{HNMR}$ (500 MHz, $\left.\mathrm{CDCl}_{3}\right) \delta 7.66(\mathrm{~m}, 4 \mathrm{H}), 7.61(\mathrm{~s}, 1 \mathrm{H}), 7.40(\mathrm{~m}, 6 \mathrm{H}), 5.43(\mathrm{~s}, 1 \mathrm{H}), 4.75(\mathrm{~s}, 2 \mathrm{H}), 4.71$ (s, 1H), $4.69(\mathrm{~s}, 1 \mathrm{H}), 4.14(\mathrm{dd}, J=12.3,4.8 \mathrm{~Hz}, 1 \mathrm{H}), 4.01(\mathrm{~m}, 1 \mathrm{H}), 3.87(\mathrm{~m}, 2 \mathrm{H}), 3.75(\mathrm{~m}, 2 \mathrm{H}), 3.68$ (m, 1H), $2.44(\mathrm{~m}, 1 \mathrm{H}), 2.34$ (ddd, $J=13.4,3.7,3.7 \mathrm{~Hz}, 2 \mathrm{H}), 2.11(\mathrm{ddd}, J=14.1,8.9,5.6 \mathrm{~Hz}, 1 \mathrm{H})$, $1.99(\mathrm{~m}, 2 \mathrm{H}), 1.86(\mathrm{~m}, 1 \mathrm{H}), 1.76(\mathrm{~m}, 1 \mathrm{H}), 1.67(\mathrm{~m}, 1 \mathrm{H}), 1.51(\mathrm{~m}, 2 \mathrm{H}), 1.05(\mathrm{~s}, 9 \mathrm{H}) ;{ }^{13} \mathrm{CNMR}(125$ $\left.\mathrm{MHz}, \mathrm{CDCl}_{3}\right) \delta 163.1,141.8,139.0,136.5,135.5,133.8,129.7,129.6,127.63,127.62,127.60$, $127.58,110.4,95.9,74.0,68.9,67.8,66.8,60.6,57.6,39.7,39.6,39.0,36.4,30.7,26.8,19.1$; high resolution mass spectrum $\left(\mathrm{ES}^{+}\right) \mathrm{m} / \mathrm{z} 600.2766\left[(\mathrm{M}+\mathrm{Na})^{+}\right.$; calcd for $\mathrm{C}_{33} \mathrm{H}_{43} \mathrm{NO}_{6} \mathrm{SiNa}$ : $600.2757]$.

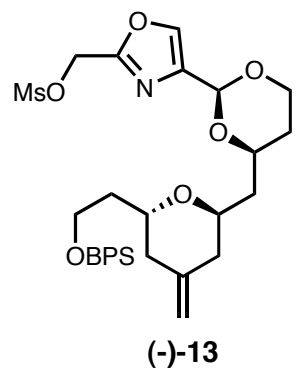

Mesylate Tricycle (-)-13: N,N-Diisopropylethylamine $(0.65 \mathrm{~mL}, 3.75 \mathrm{mmol})$ followed by dropwise addition of methanesulfonyl chloride $(0.17 \mathrm{~mL}, 2.25 \mathrm{mmol})$ were added to a solution of alcohol tricycle (-)-S $\mathrm{S}_{2}(1.08 \mathrm{~g}, 1.87 \mathrm{mmol})$ in $\mathrm{CH}_{2} \mathrm{Cl}_{2}(117 \mathrm{~mL})$ at $0{ }^{\circ} \mathrm{C}$ under an argon atmosphere. After one hour, the reaction mixture was filtered through a plug of silica gel (40\% EtOAc/hexanes) to afford mesylate tricycle (-)-13 $\left(1.20 \mathrm{~g}, 98 \%\right.$ yield) as a colorless oil: $[\alpha]_{\mathrm{D}}^{20}-15.2\left(c 1.0, \mathrm{CHCl}_{3}\right)$; IR (neat) 3071 (w), 2933 (s), 2857 (s), 1653 (w), 1587 (w), 1472 (w), 1428 (m), 1353 (s), 1238 (w), 1177 (s), 1112 (s), $1034(\mathrm{~m}), 958(\mathrm{~m}), 822$ (w), 743 (w), 704 (s), $613(\mathrm{~m}) \mathrm{cm}^{-1}$; ${ }^{1}$ HNMR (500 
$\left.\mathrm{MHz}, \mathrm{CDCl}_{3}\right) \delta 7.70(\mathrm{~s}, 1 \mathrm{H}), 7.66(\mathrm{~m}, 4 \mathrm{H}), 7.39(\mathrm{~m}, 6 \mathrm{H}), 5.43(\mathrm{~s}, 1 \mathrm{H}), 5.25(\mathrm{~s}, 2 \mathrm{H}), 4.75(\mathrm{br} \mathrm{s}, 2 \mathrm{H})$, $4.14(\mathrm{dd}, J=12.3,4.8 \mathrm{~Hz}, 1 \mathrm{H}), 4.01(\mathrm{~m}, 1 \mathrm{H}), 3.87(\mathrm{~m}, 2 \mathrm{H}), 3.72(\mathrm{~m}, 3 \mathrm{H}), 3.06(\mathrm{~s}, 3 \mathrm{H}), 2.35(\mathrm{dd}, J$ $=13.0,3.7 \mathrm{~Hz}, 2 \mathrm{H}), 2.10(\mathrm{ddd}, J=14.1,8.9,5.6 \mathrm{~Hz}, 1 \mathrm{H}), 2.00(\mathrm{~m}, 2 \mathrm{H}), 1.85$ (dddd, $J=14.1,8.6$, 5.6, $5.6 \mathrm{~Hz}, 1 \mathrm{H}), 1.71(\mathrm{~m}, 1 \mathrm{H}), 1.67(\mathrm{~m}, 1 \mathrm{H}), 1.52(\mathrm{~m}, 2 \mathrm{H}), 1.05(\mathrm{~s}, 9 \mathrm{H}) ;{ }^{13} \mathrm{CNMR}(125 \mathrm{MHz}$, $\left.\mathrm{CDCl}_{3}\right) \delta 156.7,141.7,140.0,138.0,135.5,133.8,129.7,129.6,127.65,127.64,127.61,127.60$, $110.5,95.7,74.1,68.9,67.8,66.8,61.7,60.6,39.7,39.6,39.0,38.3,36.4,30.7,26.8,19.1$; high resolution mass spectrum (ES+) $\mathrm{m} / z$ 678.2509 [(M+Na) ${ }^{+}$; calcd for $\mathrm{C}_{34} \mathrm{H}_{45} \mathrm{NO}_{8} \mathrm{SSiNa:}$ 678.2533].

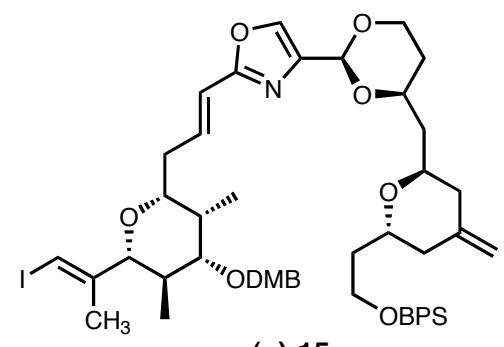

$(+)-15$

C(19-20) E-Olefin (+)-15: Tri- $n$-butylphosphine $(0.56 \mathrm{~mL}, 2.24 \mathrm{mmol})$ was added dropwise to a stirred solution of mesylate tricycle $(-)-13(0.37 \mathrm{~g}, 0.56 \mathrm{mmol})$ in anhydrous N,Ndimethylformamide (DMF) $(119 \mathrm{~mL})$ at room temperature under an argon atmosphere. After stirring for twenty-four hours, a solution of aldehyde $(+)-14(0.27 \mathrm{~g}, 0.56 \mathrm{mmol})$ in DMF $(63 \mathrm{~mL})$ was introduced dropwise via cannula. Following five minutes of stirring, 1,8diazabicyclo[5.4.0]undec-7-ene (DBU) $(0.17 \mathrm{~mL}, 1.12 \mathrm{mmol})$ was added dropwise via syringe. After one hour, the reaction was diluted with diethyl ether $(50 \mathrm{~mL})$, poured into $\mathrm{H}_{2} \mathrm{O}(50 \mathrm{~mL})$, and the organic layer was washed with $\mathrm{H}_{2} \mathrm{O}(20 \mathrm{~mL}, 5 \mathrm{x})$. The combined organic extracts were washed with saturated aqueous sodium chloride $(20 \mathrm{~mL}, 1 \mathrm{x})$, dried over $\mathrm{MgSO}_{4}$, filtered, and concentrated under reduced pressure. Purification via silica gel chromatography (25\% EtOAc/hexanes) afforded C(19-20) E-olefin (+)-15 (0.53 g, 91\% yield, 19:1, E:Z) as a light yellow oil: $[\alpha]_{D}^{20}+22.2\left(c 1.0, \mathrm{CHCl}_{3}\right)$; IR (neat) $3070(\mathrm{~m}), 2928(\mathrm{~s}), 2855(\mathrm{~s}), 1653(\mathrm{~m}), 1592(\mathrm{~m}), 1516$ (s), 1463 (s), 1427 (s), 1388 (w), $1263(\mathrm{~m}), 1238(\mathrm{~m}), 1139(\mathrm{~m}), 1110(\mathrm{~s}), 1031(\mathrm{~s}), 972(\mathrm{~m}), 893$ (w), 857 (w), $822(w), 756$ (s), 704 (s), $614(\mathrm{~m}) \mathrm{cm}^{-1} ;{ }^{1} \mathrm{HNMR}\left(500 \mathrm{MHz}, \mathrm{CDCl}_{3}\right) \delta 7.66(\mathrm{~m}, 4 \mathrm{H})$, 
$7.54(\mathrm{~s}, 1 \mathrm{H}), 7.39(\mathrm{~m}, 6 \mathrm{H}), 6.89(\mathrm{~s}, 1 \mathrm{H}), 6.87(\mathrm{~d}, J=8.3 \mathrm{~Hz}, 1 \mathrm{H}), 6.83(\mathrm{~d}, J=8.1 \mathrm{~Hz}, 1 \mathrm{H}), 6.68(\mathrm{~m}$, 1H), $6.34(\mathrm{~d}, J=16.4 \mathrm{~Hz}, 1 \mathrm{H}), 6.23(\mathrm{~s}, 1 \mathrm{H}), 5.43(\mathrm{~s}, 1 \mathrm{H}), 4.75$ (br s, 2H), 4.57 (d, J = 11.2 Hz, 1H), $4.28(\mathrm{~d}, J=11.2 \mathrm{~Hz}, 1 \mathrm{H}), 4.14(\mathrm{dd}, J=11.5,4.8 \mathrm{~Hz}, 1 \mathrm{H}), 3.99(\mathrm{~m}, 1 \mathrm{H}), 3.87(\mathrm{~s}, 3 \mathrm{H}), 3.86(\mathrm{~s}$, 3H), $3.73(\mathrm{~m}, 3 \mathrm{H}), 3.51(\mathrm{~d}, J=10.4 \mathrm{~Hz}, 1 \mathrm{H}), 3.47(\mathrm{~m}, 1 \mathrm{H}), 3.14(\mathrm{dd}, J=10.1,4.5 \mathrm{~Hz}, 1 \mathrm{H}), 2.56$ (ddd, $J=14.5,6.3,6.3 \mathrm{~Hz}, 1 \mathrm{H}), 2.35(\mathrm{~m}, 3 \mathrm{H}), 2.11(\mathrm{~m}, 2 \mathrm{H}), 2.00(\mathrm{~m}, 2 \mathrm{H}), 1.82(\mathrm{~s}, 3 \mathrm{H}), 1.80(\mathrm{~m}$, 2H), $1.68(\mathrm{~m}, 1 \mathrm{H}), 1.51(\mathrm{~m}, 2 \mathrm{H}), 1.26(\mathrm{~s}, 3 \mathrm{H}), 1.05(\mathrm{~s}, 9 \mathrm{H}), 0.97(\mathrm{~d}, J=6.7 \mathrm{~Hz}, 3 \mathrm{H}), 0.80(\mathrm{~d}, J=$ $6.3 \mathrm{~Hz}, 3 \mathrm{H}) ;{ }^{13} \mathrm{CNMR}\left(125 \mathrm{MHz}, \mathrm{CDCl}_{3}\right) \delta 161.1,149.0,148.6,146.2,141.8,139.8,135.9,135.5$, $135.3,133.8,131.0,129.6,127.64,127.63,127.61,127.60,120.2,118.4,111.1,110.9,110.4$, $96.2,87.5,82.8,81.0,77.4,77.2,74.0,69.9,68.9,67.8,66.8,60.7,55.9,55.8,39.7,39.6,39.1$, $36.4,36.1,33.5,33.3,30.7,29.6,26.8,19.1,13.5,5.7$; high resolution mass spectrum $\left(\mathrm{ES}^{+}\right) \mathrm{m} / \mathrm{z}$ 1032.3949 [(M+H) ${ }^{+}$; calcd for $\left.\mathrm{C}_{54} \mathrm{H}_{70} \mathrm{INO}_{9} \mathrm{SiH}: 1032.3943\right]$.

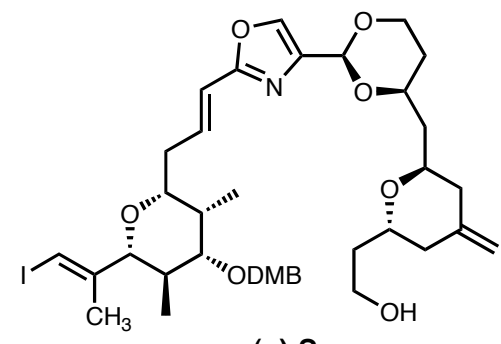

$(+)-S_{3}$

Primary Alcohol (+)-S $\mathbf{S}_{3}$ : Tetrabutylammonium fluoride (TBAF) (1.0 M/THF, $0.22 \mathrm{~mL}$, $0.22 \mathrm{mmol})$ was added dropwise via syringe to a solution of $(+)-15(0.21 \mathrm{~g}, 0.20 \mathrm{mmol})$ in freshly distilled tetrahydrofuran (THF) $(2 \mathrm{~mL})$ at room temperature under an argon atmosphere. After two hours, the reaction was quenched via dropwise addition of saturated aqueous sodium chloride $(10 \mathrm{~mL})$. The layers of the biphasic solution were separated and the aqueous layer was extracted with EtOAc $(7 \mathrm{~mL}, 4 \mathrm{x})$. The combined organic extracts were dried over $\mathrm{MgSO}_{4}$, filtered, and concentrated under reduced pressure. Purification via silica gel chromatography $(80 \%$ EtOAc/hexanes) afforded primary alcohol $(+)-S_{3}(0.14 \mathrm{~g}, 89 \%$ yield $)$ as a light yellow oil: $[\alpha]_{\mathrm{D}}^{20}$ +37.6 (c 1.0, $\mathrm{CHCl}_{3}$ ); IR (neat) 3407 (b), 3071 (w), 2940 (s), 2855 (s), 1659 (m), 1594 (w), 1516 (s), $1463(\mathrm{~m}), 1420(\mathrm{w}), 1375(\mathrm{w}), 1263(\mathrm{~m}), 1238(\mathrm{~m}), 1139(\mathrm{~m}), 1102(\mathrm{~s}), 1030(\mathrm{~s}), 973(\mathrm{~m})$, 
808 (w), 754 (s), 668 (w) cm ${ }^{-1} ;{ }^{1} \mathrm{HNMR}\left(500 \mathrm{MHz}, \mathrm{CDCl}_{3}\right.$ ) $\delta 7.60(\mathrm{~s}, 1 \mathrm{H}), 6.86(\mathrm{~m}, 3 \mathrm{H}), 6.68(\mathrm{~m}$, 1H), $6.34(\mathrm{~d}, J=16.0 \mathrm{~Hz}, 1 \mathrm{H}), 6.22(\mathrm{~d}, J=1.1 \mathrm{~Hz}, 1 \mathrm{H}), 5.56(\mathrm{~s}, 1 \mathrm{H}), 4.79(\mathrm{~s}, 1 \mathrm{H}), 4.74(\mathrm{~s}, 1 \mathrm{H})$, $4.56(\mathrm{~d}, J=11.5 \mathrm{~Hz}, 1 \mathrm{H}), 4.27(\mathrm{~d}, J=11.2 \mathrm{~Hz}, 1 \mathrm{H}), 4.24(\mathrm{dd}, J=11.1,3.6 \mathrm{~Hz}, 1 \mathrm{H}), 4.10(\mathrm{~m}, 1 \mathrm{H})$, $4.01(\mathrm{~m}, 1 \mathrm{H}), 3.94(\mathrm{~m}, 2 \mathrm{H}), 3.87(\mathrm{~s}, 3 \mathrm{H}), 3.87(\mathrm{~s}, 3 \mathrm{H}), 3.74(\mathrm{~m}, 2 \mathrm{H}), 3.5(\mathrm{~d}, J=10.4 \mathrm{~Hz}, 1 \mathrm{H}), 3.46$ (ddd, $J=7.1,7.1,1.9 \mathrm{~Hz}, 1 \mathrm{H}$ ), $3.14(\mathrm{dd}, J=10.4,4.5 \mathrm{~Hz}, 1 \mathrm{H}), 2.75(\mathrm{br} \mathrm{s}, 1 \mathrm{H}), 2.56(\mathrm{~m}, 1 \mathrm{H}), 2.42$ (dd, $J=13.0,4.8 \mathrm{~Hz}, 1 \mathrm{H}), 2.36(\mathrm{~m}, 1 \mathrm{H}), 2.29$ (dd, $J=13.0,4.1 \mathrm{~Hz}, 1 \mathrm{H}), 2.20$ (ddd, $J=14.9,8.9$, $6.3 \mathrm{~Hz}, 1 \mathrm{H}), 2.11(\mathrm{~m}, 1 \mathrm{H}), 2.04(\mathrm{~m}, 3 \mathrm{H}), 1.82(\mathrm{~m}, 3 \mathrm{H}), 1.81(\mathrm{~d}, J=1.1 \mathrm{~Hz}, 3 \mathrm{H}), 1.61(\mathrm{~m}, 1 \mathrm{H}), 1.54$ (m, 1H), $0.96(\mathrm{~d}, J=7.1 \mathrm{~Hz}, 3 \mathrm{H}), 0.79(\mathrm{~d}, J=6.3 \mathrm{~Hz}, 3 \mathrm{H}) ;{ }^{13} \mathrm{CNMR}\left(125 \mathrm{MHz}, \mathrm{CDCl}_{3}\right) \delta 161.2$, 149.0, 148.6, 146.2, 141.3, 139.7, 136.1, 135.4, 131.0, 120.2, 118.3, 111.1, 110.9, 110.7, 92.3, $87.5,82.8,80.9,77.3,74.7,70.9,69.8,69.4,66.9,60.5,55.9,55.8,40.1,39.1,38.2,36.3,36.1$, 33.6, 33.3, 31.1, 19.1, 13.5, 5.6; high resolution mass spectrum $\left(\mathrm{ES}^{+}\right) \mathrm{m} / \mathrm{z} 816.2545\left[(\mathrm{M}+\mathrm{Na})^{+}\right.$; calcd for $\left.\mathrm{C}_{38} \mathrm{H}_{52} \mathrm{INO}_{9} \mathrm{Na:} 816.2585\right]$.

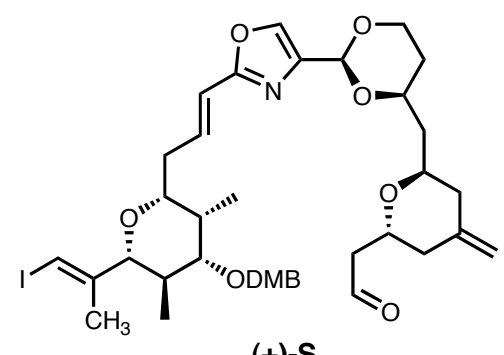

$(+)-S_{4}$

Aldehyde (+)-S: Solid sodium bicarbonate $(0.02 \mathrm{~g}, 0.20 \mathrm{mmol})$ followed by the DessMartin periodinane $(0.17 \mathrm{~g}, 0.40 \mathrm{mmol})$ were added to a solution of primary alcohol $(+)-\mathrm{S}_{3}(0.158$ g, $0.20 \mathrm{mmol})$ in $\mathrm{CH}_{2} \mathrm{Cl}_{2}(75 \mathrm{~mL})$ at $0{ }^{\circ} \mathrm{C}$ under an argon atmosphere. After stirring for ten minutes, the reaction was warmed to room temperature and after one hour and thirty minutes, the reaction was quenched via dropwise addition of saturated aqueous sodium bicarbonate $(15 \mathrm{~mL})$. The layers of the biphasic mixture were separated and the aqueous layer was extracted with $\mathrm{CH}_{2} \mathrm{Cl}_{2}$ (15 $\left.\mathrm{mL}, 4 \mathrm{x}\right)$, dried over $\mathrm{MgSO}_{4}$, filtered, and concentrated under reduced pressure. Purification via silica gel chromatography (70\% EtOAc/hexanes) afforded aldehyde (+)-S $\mathbf{S}_{4}(0.135$ g, 86\% yield): $[\alpha]_{D}^{20}+36.5\left(c 1.0, \mathrm{CHCl}_{3}\right) ;$ IR (neat) $3151(w), 3071(w), 2934(\mathrm{~s}), 2853(\mathrm{~s}), 2726$ 
(w), $1724(\mathrm{~s}), 1659(\mathrm{~m}), 1593(\mathrm{~m}), 1516(\mathrm{~s}), 1462(\mathrm{~m}), 1420(\mathrm{~m}), 1375(\mathrm{~m}), 1263(\mathrm{~s}), 1238(\mathrm{~s})$, 1156 (w), 1138 (s), 1101 (s), 1029 (s), 973 (m), 912 (m), 808 (w), 766 (w), 731 (s) cm ${ }^{-1}$; ${ }^{1}$ HNMR (500 MHz, $\left.\mathrm{CDCl}_{3}\right) \delta 9.76(\mathrm{dd}, J=3.0,1.5 \mathrm{~Hz}, 1 \mathrm{H}), 7.60(\mathrm{~s}, 1 \mathrm{H}), 6.85(\mathrm{~m}, 3 \mathrm{H}), 6.67(\mathrm{~m}, 1 \mathrm{H}), 6.33$ (d, $J=16.0 \mathrm{~Hz}, 1 \mathrm{H}), 6.22(\mathrm{~d}, J=1.1 \mathrm{~Hz}, 1 \mathrm{H}), 5.56(\mathrm{~s}, 1 \mathrm{H}), 4.8(\mathrm{~s}, 2 \mathrm{H}), 4.56(\mathrm{~d}, J=11.2 \mathrm{~Hz}, 1 \mathrm{H})$, 4.40 (dddd, $J=8.9,7.1,4.5,4.5 \mathrm{~Hz}, 1 \mathrm{H}), 4.27(\mathrm{~d}, J=11.2 \mathrm{~Hz}, 1 \mathrm{H}), 4.23(\mathrm{dd}, J=11.1,3.6 \mathrm{~Hz}$, 1H), $3.96(\mathrm{~m}, 3 \mathrm{H}), 3.87(\mathrm{~s}, 3 \mathrm{H}), 3.86(\mathrm{~s}, 3 \mathrm{H}), 3.50(\mathrm{~d}, J=10.4 \mathrm{~Hz}, 1 \mathrm{H}), 3.46$ (ddd, $J=7.1,7.1,1.9$ Hz, 1H), $3.14(\mathrm{dd}, J=10.4,4.8 \mathrm{~Hz}, 1 \mathrm{H}), 2.70(\mathrm{~m}, 1 \mathrm{H}), 2.53(\mathrm{~m}, 1 \mathrm{H}), 2.47(\mathrm{dd}, J=16.4,4.8 \mathrm{~Hz}$, 1H), 2.37 (m, 3H), 2.15 (ddd, $J=14.5,9.3,5.6 \mathrm{~Hz}, 1 \mathrm{H}), 2.10$ (ddd, $J=6.7,4.8,1.5 \mathrm{~Hz}, 1 \mathrm{H}$ ), 2.03 (m, 2H), $1.80(\mathrm{~d}, J=1.1 \mathrm{~Hz}, 3 \mathrm{H}), 1.77(\mathrm{~m}, 1 \mathrm{H}), 1.56(\mathrm{~m}, 2 \mathrm{H}), 1.25(\operatorname{app~t}, J=7.1 \mathrm{~Hz}, 1 \mathrm{H}), 0.96(\mathrm{~d}$, $J=7.1 \mathrm{~Hz}, 3 \mathrm{H}), 0.79(\mathrm{~d}, J=6.3 \mathrm{~Hz}, 3 \mathrm{H}) ;{ }^{13} \mathrm{CNMR}\left(125 \mathrm{MHz}, \mathrm{CDCl}_{3}\right) \delta 200.5,161.2,149.0,148.6$, $146.2,140.5,139.8,136.0,135.3,130.9,120.2,118.4,111.4,111.1,110.9,96.3,87.5,82.8$, $80.9,77.3,74.0,69.9,68.8,67.2,66.9,55.9,55.8,47.1,39.4,39.2,38.8,36.1,33.5,33.3,30.8$ $19.1,13.5,5.7$; high resolution mass spectrum $\left(\mathrm{ES}^{+}\right) \mathrm{m} / \mathrm{z} 814.2392\left[(\mathrm{M}+\mathrm{Na})^{+}\right.$; calcd for $\mathrm{C}_{38} \mathrm{H}_{50} \mathrm{NNO}_{9} \mathrm{Na}:$ 814.2428].

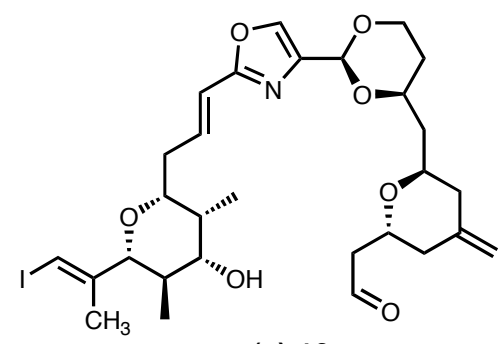

$(+)-16$

Secondary Alcohol-Aldehyde (+)-16: 2,3-Dichloro-5,6-dicyano-1,4-benzoquinone (DDQ) $(0.079 \mathrm{~g}, 0.35 \mathrm{mmol})$ and $\mathrm{pH} 7$ phosphate buffer $(5.7 \mathrm{~mL})$ were added to a solution of aldehyde $(+)-\mathrm{S}_{4}(0.137 \mathrm{~g}, 0.17 \mathrm{mmol})$ in $\mathrm{CH}_{2} \mathrm{Cl}_{2}(24 \mathrm{~mL})$ at room temperature under an argon atmosphere. After three hours and thirty minutes, the reaction mixture was poured into saturated aqueous sodium bicarbonate $(20 \mathrm{~mL})$. The layers of the biphasic mixture were separated and the aqueous layer was extracted with $\mathrm{CH}_{2} \mathrm{Cl}_{2}(15 \mathrm{~mL}, 4 \mathrm{x})$. The combined organic extracts were dried over $\mathrm{MgSO}_{4}$, filtered, and concentrated under reduced pressure. Purification via silica gel 
chromatography (80\% EtOAc/hexanes) afforded secondary alcohol-aldehyde (+)-16 (0.108 g, 98\% yield) as a light yellow oil: $[\alpha]_{\mathrm{D}}^{20}+34.7\left(c 1.0, \mathrm{CHCl}_{3}\right)$; IR (neat) $3420(b), 3151(w), 3071(w)$, 2924 (s), 2855 (s), 2726 (w), 1718 (s), 1654 (s), 1618 (w), $1541(\mathrm{~m}), 1457$ (m), 1279 (w), 1100 (s), 1012 (s), 892 (w), 755 (m), 671 (w) cm ${ }^{-1} ;{ }^{1} \mathrm{HNMR}$ (500 MHz, $\mathrm{CDCl}_{3}$ ) 89.76 (dd, J = 3.0, 1.5 $\mathrm{Hz}, 1 \mathrm{H}), 7.59(\mathrm{~s}, 1 \mathrm{H}), 6.66(\mathrm{~m}, 1 \mathrm{H}), 6.33(\mathrm{~d}, J=16.0 \mathrm{~Hz}, 1 \mathrm{H}), 6.23(\mathrm{~d}, J=1.1 \mathrm{~Hz}, 1 \mathrm{H}), 5.55(\mathrm{~s}$, 1H), $4.80(\mathrm{~s}, 2 \mathrm{H}), 4.40(\mathrm{~m}, 1 \mathrm{H}), 4.23(\mathrm{dd}, J=11.2,4.8 \mathrm{~Hz}, 1 \mathrm{H}), 3.95(\mathrm{~m}, 3 \mathrm{H}), 3.51(\mathrm{~m}, 1 \mathrm{H}), 3.50$ (d, $J=10.2 \mathrm{~Hz}, 1 \mathrm{H}), 3.43(\mathrm{dd}, J=10.4,4.5 \mathrm{~Hz}, 1 \mathrm{H}), 2.70(\mathrm{~m}, 1 \mathrm{H}), 2.54(\mathrm{~m}, 1 \mathrm{H}), 2.50(\mathrm{dd}, J=$ 16.4, 4.8, Hz, 1H), 2.48 (ddd, $J=16.4,4.8,1.5 \mathrm{~Hz}, 2 \mathrm{H}$ ), $2.32(\mathrm{~m}, 1 \mathrm{H}), 2.12$ (ddd, $J=14.5,9.3$, $5.6 \mathrm{~Hz}, 1 \mathrm{H}), 2.03(\mathrm{~m}, 2 \mathrm{H}), 1.9(\mathrm{~m}, 1 \mathrm{H}), 1.81(\mathrm{~d}, J=1.1 \mathrm{~Hz}, 3 \mathrm{H}), 1.60(\mathrm{~m}, 5 \mathrm{H}), 0.95(\mathrm{~d}, J=7.1 \mathrm{~Hz}$, 3H), 0.81 (d, $J=6.7 \mathrm{~Hz}, 3 \mathrm{H}) ;{ }^{13} \mathrm{CNMR}\left(125 \mathrm{MHz}, \mathrm{CDCl}_{3}\right) \delta 200.5,161.2,146.0,140.5,139.8$, $136.0,135.3,118.3,111.4,96.3,87.3,80.9,77.6,76.3,74.0,68.7,67.2,66.9,47.6,39.4,39.2$ 38.8, 38.0, 35.9, 34.5, 30.8, 19.2, 13.1, 5.4; high resolution mass spectrum (ES+) $\mathrm{m} / \mathrm{z} 642.1911$ $\left[(\mathrm{M}+\mathrm{H})^{+}\right.$; calcd for $\left.\mathrm{C}_{29} \mathrm{H}_{40} \mathrm{INO}_{7} \mathrm{H}: 642.1928\right]$.

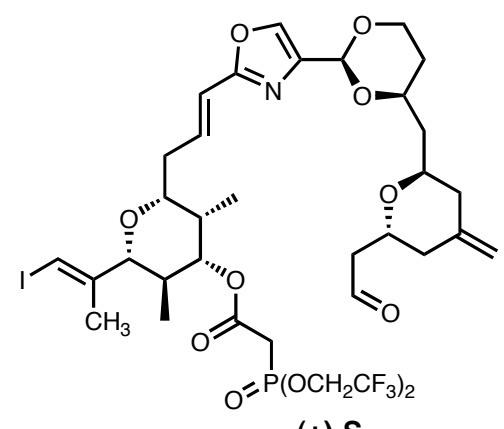

$(+)-S_{5}$

Phosphonate Ester (+)-S $\mathbf{S}_{5}$ A solution of bis-(2,2,2-trifluoroethyl)phosphonate acid 17 (0.522 g, $1.72 \mathrm{mmol})$ in $\mathrm{CH}_{2} \mathrm{Cl}_{2}(30 \mathrm{~mL})$ was added via cannula to a solution of secondary alcohol-aldehyde $(+)-16(0.221 \mathrm{~g}, 0.34 \mathrm{mmol})$ in $\mathrm{CH}_{2} \mathrm{Cl}_{2}(40 \mathrm{~mL})$ at room temperature under an argon atmosphere. After stirring for ten minutes, 1-[3-(dimethylamino)propyl]-3ethylcarbodiimide-methiodide (EDCl•Mel) $(0.511 \mathrm{~g}, 1.72 \mathrm{mmol})$ and 1-hydroxybenzotriazole (HOBT) $(0.005 \mathrm{~g}, 0.03 \mathrm{mmol})$ were added to the reaction mixture. After forty-five minutes, the reaction was filtered through a plug of silica gel (80\% EtOAc/hexanes) to afford phosphonate 
ester (+)-S $\mathbf{S}_{5}\left(0.261 \mathrm{~g}, 82 \%\right.$ yield) as a light yellow oil: $[\alpha]_{D}^{20}+17.6\left(c 1.0, \mathrm{CHCl}_{3}\right)$; IR (neat) 3077 (w), 2924 (s), $2855(m), 2726(w), 1728$ (s), $1656(w), 1624(w), 1539(w), 1419(m), 1397(w)$, 1305 (s), 1267 (s), 1173 (s), 1098 (s), 1070 (s), 1040 (m), 963 (m), 893 (m) cm ${ }^{-1}$; ${ }^{1}$ HNMR (500 $\left.\mathrm{MHz}, \mathrm{CDCl}_{3}\right) \delta 9.76(\mathrm{dd}, J=3.0,1.5 \mathrm{~Hz}, 1 \mathrm{H}), 7.59(\mathrm{~s}, 1 \mathrm{H}), 6.63(\mathrm{~m}, 1 \mathrm{H}), 6.31(\mathrm{~d}, J=16.0 \mathrm{~Hz}$, 1H), $6.28(\mathrm{~s}, 1 \mathrm{H}), 5.55(\mathrm{~s}, 1 \mathrm{H}), 4.81(\mathrm{~s}, 2 \mathrm{H}), 4.74(\mathrm{dd}, J=11.2,4.5 \mathrm{~Hz}, 1 \mathrm{H}), 4.43(\mathrm{~m}, 5 \mathrm{H}), 4.24$ (dd, $J=11.2,4.8 \mathrm{~Hz}, 1 \mathrm{H}), 3.98(\mathrm{~m}, 1 \mathrm{H}), 3.93(\mathrm{~m}, 2 \mathrm{H}), 3.58(\mathrm{~d}, J=10.1 \mathrm{~Hz}, 1 \mathrm{H}), 3.56(\mathrm{~m}, 1 \mathrm{H})$, $3.20(\mathrm{~s}, 1 \mathrm{H}), 3.16(\mathrm{~s}, 1 \mathrm{H}), 2.70(\mathrm{~m}, 1 \mathrm{H}), 2.56(\mathrm{~m}, 1 \mathrm{H}), 2.48(\mathrm{dd}, J=16.0,4.8 \mathrm{~Hz}, 1 \mathrm{H}), 2.40$ (ddd, $J$ = 13.0, 4.6, $4.5 \mathrm{~Hz}, 2 \mathrm{H}), 2.28(\mathrm{~m}, 1 \mathrm{H}), 2.16(\mathrm{ddd}, J=14.1,9.3,5.6 \mathrm{~Hz}, 1 \mathrm{H}), 2.09(\mathrm{~m}, 1 \mathrm{H}), 2.04$ (dd, $J=13.4,6.0 \mathrm{~Hz}, 2 \mathrm{H}), 1.91(\mathrm{~m}, 1 \mathrm{H}), 1.83(\mathrm{~s}, 3 \mathrm{H}), 1.79(\mathrm{~m}, 2 \mathrm{H}), 1.56(\mathrm{~m}, 1 \mathrm{H}), 0.96(\mathrm{~d}, J=7.1$ $\mathrm{Hz}, 3 \mathrm{H}), 0.72(\mathrm{~d}, J=6.7 \mathrm{~Hz}, 3 \mathrm{H}) ;{ }^{13} \mathrm{CNMR}\left(125 \mathrm{MHz}, \mathrm{CDCl}_{3}\right) \delta 200.5,163.9,161.0,145.3,140.5$, $139.8,135.4,135.3,118.6,111.4,96.3,87.1,81.7,80.5,76.7,74.0,68.7,67.2,66.9,62.7,62.4$, 47.6, 39.4, 39.2, 38.8, 35.8, 35.3, 34.6, 33.5, 32.0, 30.8, 29.6, 19.1, 12.9, 6.0; high resolution mass spectrum $\left(\mathrm{ES}^{+}\right) \mathrm{m} / \mathrm{z} 928.1787\left[(\mathrm{M}+\mathrm{H})^{+}\right.$; calcd for $\left.\mathrm{C}_{35} \mathrm{H}_{45} \mathrm{~F}_{6} \mathrm{INO}_{11} \mathrm{PH}: 928.1757\right]$.

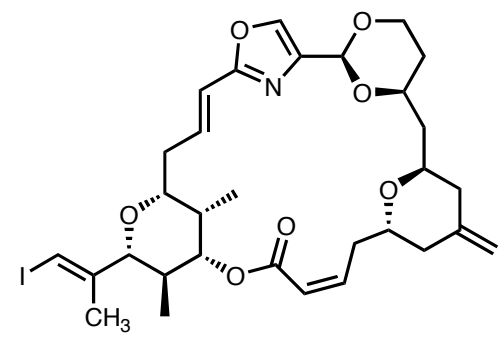

$(+)-9 Z$

Z-C(2-3) Macrocycle (+)-9Z: $18-C r o w n-6(3.98 \mathrm{~g}, 15.1 \mathrm{mmol})$ and solid potassium carbonate $(0.446 \mathrm{~g}, 3.23 \mathrm{mmol})$ were added to a flask charged with freshly distilled toluene (195 $\mathrm{mL}$ ) at room temperature under an argon atmosphere. After stirring for three hours, a solution of phosphonate ester $(+)-S_{5}(0.25 \mathrm{~g}, 0.27 \mathrm{mmol})$ in freshly distilled toluene $(170 \mathrm{~mL})$ was added dropwise via cannula and allowed to stir at room temperature. After two hours, the reaction mixture was poured into saturated aqueous sodium chloride $(100 \mathrm{~mL})$ and the layers of the resultant biphasic mixture were separated. The aqueous layer was extracted with EtOAc $(30 \mathrm{~mL}$, 4x), dried over $\mathrm{MgSO}_{4}$, filtered and concentrated under reduced pressure. Purification via silica 
gel chromatography afforded $Z-C(2-3)$ macrocycle (+)-9Z (0.082 g, 46\% yield) as an off white foam: Z-C(2-3) Macrocycle (+)-9Z: $[\alpha]_{\mathrm{D}}^{20}+25.7\left(\right.$ c 0.2, $\left.\mathrm{CHCl}_{3}\right)$; IR (neat) 2923 (s), 2843 (s), 1717 (s), $1653(\mathrm{~m}), 1557(\mathrm{w}), 1456(\mathrm{w}), 1280(\mathrm{~m}), 1192(\mathrm{~m}), 1149(\mathrm{~m}), 1091(\mathrm{~s}), 1018(\mathrm{~m}), 886(\mathrm{w}), 667$ (s) $\mathrm{cm}^{-1} ;{ }^{1} \mathrm{HNMR}\left(500 \mathrm{MHz}, \mathrm{C}_{6} \mathrm{D}_{6}\right) \delta 7.61(\mathrm{~s}, 1 \mathrm{H}), 6.92(\mathrm{~m}, 1 \mathrm{H}), 6.15(\mathrm{~d}, J=15.9 \mathrm{~Hz}, 1 \mathrm{H}), 6.11(\mathrm{~s}$, 1H), $5.87(\mathrm{dd}, J=10.2,2.0 \mathrm{~Hz}, 1 \mathrm{H}), 5.55(\mathrm{ddd}, J=10.2,10.1,3.0 \mathrm{~Hz}, 1 \mathrm{H}), 5.45(\mathrm{~d}, J=0.9 \mathrm{~Hz}$, 1H), $5.26(\mathrm{~s}, 1 \mathrm{H}), 4.85(\mathrm{~s}, 1 \mathrm{H}), 4.57(\mathrm{dd}, J=11.2,4.4 \mathrm{~Hz}, 1 \mathrm{H}), 4.40(\mathrm{~m}, 1 \mathrm{H}), 4.16(\mathrm{dd}, J=11.8$, $6.1 \mathrm{~Hz}, 1 \mathrm{H}), 4.01$ (ddd, $J=15.8,11.3,11.1 \mathrm{~Hz}, 1 \mathrm{H}), 3.91$ (dd, $J=11.7,4.4 \mathrm{~Hz}, 1 \mathrm{H}), 3.59$ (app t, $J$ $=11.1 \mathrm{~Hz}, 1 \mathrm{H}), 3.46(\mathrm{ddd}, J=12.1,11.7,2.5 \mathrm{~Hz}, 1 \mathrm{H}), 3.34(\mathrm{~d}, J=10.1 \mathrm{~Hz}, 1 \mathrm{H}), 3.34(\mathrm{~m}, 1 \mathrm{H})$, $2.91(\mathrm{~d}, J=12.2 \mathrm{~Hz}, 1 \mathrm{H}), 2.67(\operatorname{app} t, J=6.3 \mathrm{~Hz}, 1 \mathrm{H}), 2.51(\mathrm{~m}, 2 \mathrm{H}), 2.36(\mathrm{~m}, 2 \mathrm{H}), 2.09(\mathrm{~m}, 1 \mathrm{H})$, $2.08(\mathrm{~d}, J=12.7 \mathrm{~Hz}, 1 \mathrm{H}), 2.02(\mathrm{~d}, J=11.2 \mathrm{~Hz}, 1 \mathrm{H}), 1.94(\mathrm{~m}, 1 \mathrm{H}), 1.88(\mathrm{~d}, J=0.9 \mathrm{~Hz}, 3 \mathrm{H}), 1.55$ (m, 2H), 1.02 (d, $J=6.9 \mathrm{~Hz}, 3 \mathrm{H}), 0.84(\mathrm{~d}, J=13.4 \mathrm{~Hz}, 1 \mathrm{H}), 0.68(\mathrm{~d}, J=6.5 \mathrm{~Hz}, 3 \mathrm{H}) ;{ }^{13} \mathrm{CNMR}(125$ $\left.\mathrm{MHz}, \mathrm{CDCl}_{3}\right) \delta 165.5,161.1,145.7,144.4,141.4,139.6,134.6,134.1,120.8,119.1,110.1,97.0$, $87.6,81.7,79.1,78.2,76.7,73.2,68.2,67.0,41.1,39.0,36.8,34.1,32.5,31.8,30.3,29.6,19.1$, 13.0, 5.7; high resolution mass spectrum $\left(\mathrm{ES}^{+}\right) \mathrm{m} / \mathrm{z} 688.1736\left[(\mathrm{M}+\mathrm{Na})^{+}\right.$; calcd for $\mathrm{C}_{31} \mathrm{H}_{40} \mathrm{INO}_{7} \mathrm{Na}$ : 688.1747].

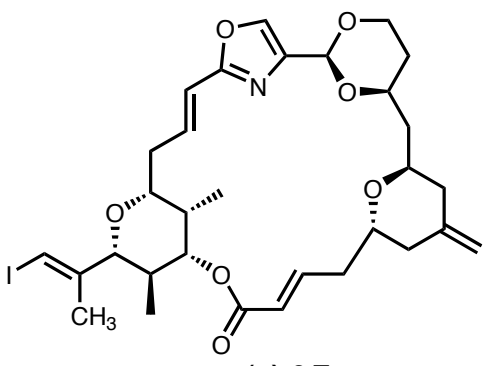

$(+)-9 E$

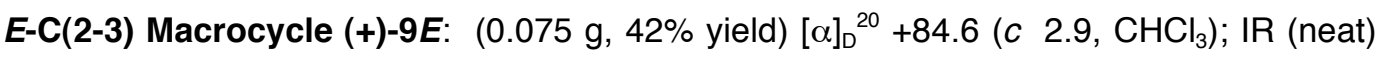
2926 (b), 2853 (b), 1720 (s), 1656 (s), 1153 (s), 1097 (s), 1010 (b), 755 (s); ${ }^{1} \mathrm{HNMR}$ (500 MHz, $\left.\mathrm{C}_{6} \mathrm{D}_{6}\right) \delta 7.32(\mathrm{~s}, 1 \mathrm{H}), 6.68(\mathrm{~m}, 1 \mathrm{H}), 6.08(\mathrm{~d}, J=16.0 \mathrm{~Hz}, 1 \mathrm{H}), 6.03(\mathrm{~d}, J=15.4 \mathrm{~Hz}, 1 \mathrm{H}), 5.98(\mathrm{~s}$, 1H), $5.34(\mathrm{~s}, 1 \mathrm{H}), 4.97(\mathrm{dd}, J=11.1,4.3 \mathrm{~Hz}, 1 \mathrm{H}), 4.72(\mathrm{~s}, 1 \mathrm{H}) ; 4.66(\mathrm{~s}, 1 \mathrm{H}), 4.18(\mathrm{~m}, 1 \mathrm{H}), 3.85$ (dd, $J=11.4,3.8 \mathrm{~Hz}, 1 \mathrm{H}), 3.36(\mathrm{~m}, 2 \mathrm{H}), 3.23(\mathrm{~m}, 2 \mathrm{H}), 3.17(\mathrm{app} \mathrm{t}, J=9.9 \mathrm{~Hz}, 1 \mathrm{H}), 2.25(\mathrm{~m}, 2 \mathrm{H})$, $2.11(\mathrm{~m}, 3 \mathrm{H}), 1.91(\mathrm{dd}, J=13.0,3.1 \mathrm{~Hz}, 1 \mathrm{H}), 1.83(\mathrm{~m}, 2 \mathrm{H}), 1.76(\mathrm{~s}, 3 \mathrm{H}), 1.69(\mathrm{~m}, 4 \mathrm{H}), 0.92(\mathrm{app} \mathrm{t}$, 
$J=7.1 \mathrm{~Hz}, 1 \mathrm{H}), 0.88(\operatorname{app~t}, J=7.0 \mathrm{~Hz}, 1 \mathrm{H}), 0.86(\mathrm{~d}, J=6.7 \mathrm{~Hz}, 3 \mathrm{H}), 0.75(\operatorname{app~t}, J=7.1 \mathrm{~Hz}, 1 \mathrm{H})$, $0.67(\mathrm{~d}, J=6.5 \mathrm{~Hz}, 3 \mathrm{H}) ;{ }^{13} \mathrm{CNMR}\left(125 \mathrm{MHz}, \mathrm{C}_{6} \mathrm{H}_{6}\right) \delta 166.8,161.9,147.0,146.6,142.2,141.1$, 135.6, 123.8, 119.1, 111.2, 95.6, 87.8, 81.2, 78.6, 77.4, 73.6, 70.5, 69.6, 66.6, 40.9, 40.7, 38.6, 38.2 , 34.7, 32.6, 31.9, 30.4, 19.4, 13.2, 6.2, 1.5; high resolution mass spectrum (ES $\left.{ }^{+}\right) \mathrm{m} / \mathrm{z}$ $688.0859\left[(\mathrm{M}+\mathrm{Na})^{+}\right.$; calcd for $\mathrm{C}_{31} \mathrm{H}_{40} \mathrm{INO}_{7} \mathrm{Na:}$ 688.0850].

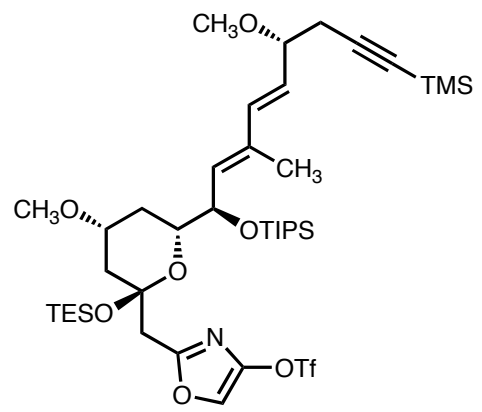

$(-)-S_{6}$

C(33)-TES Acetal Sidechain (-)-S $\mathbf{S}_{6}$ : Oxazole $19(0.74 \mathrm{~g}, 2.38 \mathrm{mmol})$ was added to a solution of dienyl lactone $(-)-18(0.255 \mathrm{~g}, 0.47 \mathrm{mmol})$ in freshly distilled tetrahydrofuran (THF) $(22.4 \mathrm{~mL})$ at room temperature under an argon atmosphere. After cooling to $0{ }^{\circ} \mathrm{C}$, isopropylmagnesium chloride $(i-\mathrm{PrMgCl})(2.0 \mathrm{M} / \mathrm{THF}, 0.59 \mathrm{~mL}, 1.20 \mathrm{mmol})$ was added dropwise over thirty minutes followed by stirring for twenty minutes. Additional $i-\mathrm{PrMgCl}(2.0 \mathrm{M} / \mathrm{THF}, 0.59 \mathrm{~mL}$, $1.20 \mathrm{mmol}$ ) was added dropwise over thirty minutes and allowed to stir for twenty minutes. A final addition of $i-\mathrm{PrMgCl}(2.0 \mathrm{M} / \mathrm{THF}, 0.59 \mathrm{~mL}, 1.20 \mathrm{mmol})$ was added dropwise over thirty minutes and after stirring for twenty minutes, the reaction was quenched via dropwise addition of saturated aqueous sodium bicarbonate $(10 \mathrm{~mL})$. The layers of the biphasic solution were separated and the aqueous layer was extracted with EtOAc $(10 \mathrm{~mL}, 3 x)$. The combined organic extracts were dried over $\mathrm{MgSO}_{4}$, filtered, and concentrated under reduced pressure. The resultant light orange oil was filtered through a plug of silica gel (30\% EtOAc/hexanes) to afford the corresponding hemi-acetal, which was immediately dissolved in freshly distilled diethyl ether $(1.4 \mathrm{~mL})$ and anhydrous acetonitrile $(1.0 \mathrm{~mL})$. After cooling to $-78^{\circ} \mathrm{C}$ under an argon atmosphere, 2,6-lutidine $(0.425 \mathrm{~mL}, 3.66 \mathrm{mmol})$ was added followed by dropwise addition of triethylsilyl 
trifluoromethanesulfonate (TESOTf) $(0.278 \mathrm{~mL}, 1.22 \mathrm{mmol})$. After twenty-four hours, the reaction was quenched via dropwise addition of saturated aqueous sodium bicarbonate $(3 \mathrm{~mL})$ and allowed to warm to room temperature. The layers of the biphasic solution were separated and the aqueous layer was extracted with $\mathrm{CH}_{2} \mathrm{Cl}_{2}(5 \mathrm{~mL}, 3 \mathrm{x})$. The combined organic extracts were dried over $\mathrm{MgSO}_{4}$, filtered, and concentrated under reduced pressure. Purification via silica gel chromatography (5\% EtOAc/hexanes) afforded C(33)-TES acetal sidechain (-)-S 6 (0.224 g, 54\% yield, 2 steps) as a colorless oil: $[\alpha]_{D}^{20}-8.4\left(c 0.1, \mathrm{CHCl}_{3}\right)$; IR (neat) 2958 (b), 2868 (b), 2179 (s), 1591 (s), 1434 (s), 1231 (b), 1139 (s), 1094 (s), 1000 (s), 853 (s), 606 (s); ${ }^{1} \mathrm{HNMR}$ (500 MHz, $\left.\mathrm{C}_{6} \mathrm{D}_{6}\right) \delta 7.14(\mathrm{~s}, 1 \mathrm{H}), 6.25(\mathrm{~d}, J=15.7 \mathrm{~Hz}, 1 \mathrm{H}), 5.73(\mathrm{dd}, J=15.7,7.3 \mathrm{~Hz}, 1 \mathrm{H}), 5.53(\mathrm{~d}, J=8.9$ $\mathrm{Hz}, 1 \mathrm{H}), 4.83(\mathrm{dd}, J=8.9,4.9 \mathrm{~Hz}, 1 \mathrm{H}), 4.09(\mathrm{~m}, 1 \mathrm{H}), 3.71(\mathrm{~m}, 1 \mathrm{H}), 3.66(\mathrm{dd}, J=9.9,4.2 \mathrm{~Hz}, 1 \mathrm{H})$, $3.27(\mathrm{dd}, J=13.9,7.0 \mathrm{~Hz}, 1 \mathrm{H}), 3.15(\mathrm{~s}, 3 \mathrm{H}), 3.13(\mathrm{~s}, 3 \mathrm{H}), 2.87(\mathrm{~d}, J=14.2 \mathrm{~Hz}, 1 \mathrm{H}), 2.79(\mathrm{~d}, J=$ 14.3 Hz, 1H), $2.55(\mathrm{dd}, J=16.8,5.3 \mathrm{~Hz}, 1 \mathrm{H}), 2.43(\mathrm{dd}, J=16.7,6.7 \mathrm{~Hz}, 1 \mathrm{H}), 2.37(\mathrm{dd}, J=10.3$, $2.0 \mathrm{~Hz}, 1 \mathrm{H}$ ), 2.26 (ddd, $J=14.0,4.1,1.4 \mathrm{~Hz}, 1 \mathrm{H}), 1.83(\mathrm{~s}, 3 \mathrm{H}), 1.66($ app t, $J=11.4 \mathrm{~Hz}, 1 \mathrm{H}), 1.12$ (m, 21H), $1.00(\operatorname{app~t}, J=7.9 \mathrm{~Hz}, 9 \mathrm{H}), 0.65(\operatorname{app~q}, J=7.9 \mathrm{~Hz}, 6 \mathrm{H}), 0.22(\mathrm{~s}, 9 \mathrm{H}) ;{ }^{13} \mathrm{CNMR}(125$ $\left.\mathrm{MHz}, \mathrm{C}_{6} \mathrm{D}_{6}\right) \delta 159.0,144.9,137.1,134.8,132.6,104.1,99.3,86.6,80.6,74.3,73.9,70.9,65.9$, $56.4,55.1,42.3,41.4,31.4,27.1,18.3,18.2,15.5,13.5,12.8,7.2,6.6,0.22$; high resolution mass spectrum $\left(\mathrm{ES}^{+}\right) \mathrm{m} / \mathrm{z} 904.3924$ [(M+Na) ${ }^{+}$; calcd for $\mathrm{C}_{40} \mathrm{H}_{70} \mathrm{~F}_{3} \mathrm{NO}_{9} \mathrm{SSi}_{3} \mathrm{Na}$ : 904.3927].

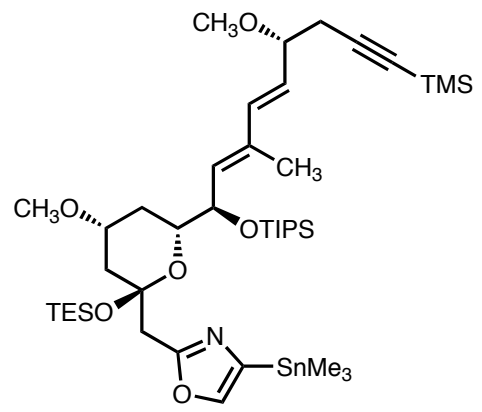

$(-)-8$

C(33)-TES Stannyl Sidechain (-)-8: C(33)-TES acetal sidechain (-)-S ( $_{6}(0.028 \mathrm{~g}, 0.033$ $\mathrm{mmol})$ was combined with hexamethylditin $\left[\left(\mathrm{Me}_{3} \mathrm{Sn}\right)_{2}\right](0.015 \mathrm{~g}, 0.046 \mathrm{mmol})$ in a sealed tube (100 $\mathrm{mL})$, azeotroped from benzene $(2 \mathrm{~mL}, 3 \mathrm{x})$, and dried under vacuum for thirty minutes. Flame 
dried lithium chloride ( $\mathrm{LiCl})(0.022 \mathrm{~g}, 0.52 \mathrm{mmol})$, tetrakis(triphenylphosphine)palladium $\left[\mathrm{Pd}\left(\mathrm{PPh}_{3}\right)_{4}\right](5.6 \mathrm{mg}, 0.005 \mathrm{mmol})$ and anhydrous dioxane $(0.50 \mathrm{~mL}$, freeze pump thawed, $3 \mathrm{x})$ were added to the tube in a glove bag, under an argon atmosphere. The tube was sealed and heated to $90{ }^{\circ} \mathrm{C}$ with stirring behind a blast shield. After thirteen hours, the reaction mixture was cooled to room temperature and concentrated under reduced pressure. The resultant dark brown slurry was purified via silica gel chromatography (5\% EtOAc/hexanes) to afford C(33)-TES stannyl sidechain (-)-8 (0.016 g, 55\% yield) as a colorless oil: $[\alpha]_{D}^{20}-31.1\left(c 0.1, \mathrm{CHCl}_{3}\right)$; IR (neat) 2957 (b), 1462 (s), 1248 (s), 1095 (b), 1000 (s), 843 (s), 743 (b); ${ }^{1} \mathrm{HNMR}$ (500 MHz, C $6 \mathrm{D}_{6}$ ) $\delta 7.36(\mathrm{~s}, 1 \mathrm{H}), 6.32(\mathrm{~d}, J=15.7 \mathrm{~Hz}, 1 \mathrm{H}), 5.75(\mathrm{dd}, J=15.7,7.3 \mathrm{~Hz}, 1 \mathrm{H}), 5.64(\mathrm{~d}, J=8.7 \mathrm{~Hz}, 1 \mathrm{H})$, $4.90(\mathrm{dd}, J=8.6,4.8 \mathrm{~Hz}, 1 \mathrm{H}), 4.18(\mathrm{dd}, J=11.9,4.7 \mathrm{~Hz}, 1 \mathrm{H}), 3.79(\mathrm{~m}, 1 \mathrm{H}), 3.69(\mathrm{dd}, J=13.1$, $6.8 \mathrm{~Hz}, 1 \mathrm{H}), 3.28(\mathrm{~d}, J=14.4 \mathrm{~Hz}, 1 \mathrm{H}), 3.23(\mathrm{~d}, J=14.3 \mathrm{~Hz}, 1 \mathrm{H}), 3.14(\mathrm{~s}, 3 \mathrm{H}), 3.13(\mathrm{~s}, 3 \mathrm{H}), 2.58$ (dd, $J=16.7,5.5 \mathrm{~Hz}, 1 \mathrm{H}$ ), 2.51 (dd, $J=12.5,4.2 \mathrm{~Hz}, 1 \mathrm{H}$ ), 2.44 (dd, $J=16.7,6.9 \mathrm{~Hz}, 1 \mathrm{H}$ ), 2.39 (d, $J=12.3 \mathrm{~Hz}, 1 \mathrm{H}), 1.90(\mathrm{~s}, 3 \mathrm{H}), 1.76(\operatorname{app~t}, J=12.2 \mathrm{~Hz}, 1 \mathrm{H}), 1.34(\mathrm{~m}, 1 \mathrm{H}), 1.16(\mathrm{~m}, 21 \mathrm{H}), 1.06$ (app t, $J=7.9 \mathrm{~Hz}, 9 \mathrm{H}), 0.76(\operatorname{app~q}, J=15.7 \mathrm{~Hz}, 6 \mathrm{H}), 0.27(\mathrm{~s}, 9 \mathrm{H}), 0.23(\mathrm{~s}, 9 \mathrm{H}) ;{ }^{13} \mathrm{CNMR}(125$ $\left.\mathrm{MHz}, \mathrm{C}_{6} \mathrm{D}_{6}\right) \delta 161.7,145.6,137.7,135.1,133.5,104.6,100.3,86.9,81.1,74.8,74.5,71.8,56.8$, $55.5,42.8,42.3,32.2,30.6,27.8,18.7,18.6,14.1,13.2,7.7,7.1,0.6,-9.3$; high resolution mass spectrum (ES $\left.{ }^{+}\right) \mathrm{m} / z$ 920.4139 [(M+Na) $)^{+}$; calcd for $\left.\mathrm{C}_{42} \mathrm{H}_{79} \mathrm{NO}_{6} \mathrm{Si}_{3} \mathrm{SnNa}: 920.4012\right]$.

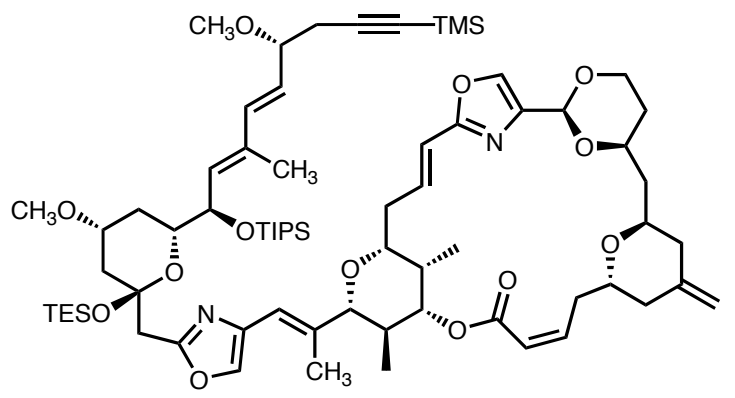

$(+)-\mathrm{S}_{7} Z$

Protected Z-C(2-3) Acetal Alkynyl Phorboxazole (+)-S $Z$ : Z-C(2-3) macrocycle (+)-9Z (3.9 mg, $0.006 \mathrm{mmol})$ was combined with C(33)-TES stannyl sidechain (-)-8 (7.8 mg, $0.009 \mathrm{mmol})$ in a flame-dried round-bottom flask $(5 \mathrm{~mL})$, azeotroped from benzene $(1 \mathrm{~mL}, 3 \mathrm{x})$ and dried under 
vacuum for two hours. Tris(dibenzylideneacetone)dipalladium-chloroform adduct $\left[\mathrm{Pd}_{2}(\mathrm{dba})_{3} \cdot \mathrm{CHCl}_{3}\right](1.2 \mathrm{mg}, 0.001 \mathrm{mmol})$, triphenylarsine $\left(\mathrm{AsPh}_{3}\right)(2.2 \mathrm{mg}, 0.007 \mathrm{mmol})$, and $\mathrm{Ph}_{2} \mathrm{PO}_{2} \mathrm{NBu}_{4}(4.1 \mathrm{mg}, 0.009 \mathrm{mmol})$ followed by DMF $(0.1 \mathrm{~mL}$, sparged with argon for thirty minutes) and $\mathrm{N}, \mathrm{N}$-diisopropylethylamine $(0.001 \mathrm{~mL}, 0.006 \mathrm{mmol})$ were added to the flask and the reaction stirred at room temperature under an argon atmosphere. After seventeen hours, the light brown reaction mixture was introduced directly onto a silica gel column (25\% EtOAc/hexanes) to afford protected $Z-C(2-3)$ acetal alkynyl phorboxazole (+)-S $\mathbf{Z}(5.2 \mathrm{mg}, 68 \%$ yield) as a light yellow oil: $[\alpha]_{D}^{20}+6.7\left(c 0.3, \mathrm{CHCl}_{3}\right)$; IR (neat) 2924 (b), 1720 (s), 1651 (w), 1463 (s), 1376 (w), 1247 (w), 1091 (s), 1018 (b), 843 (s), 742 (w); ${ }^{1} \mathrm{HNMR}\left(500 \mathrm{MHz}, \mathrm{C}_{6} \mathrm{D}_{6}\right) \delta 7.52$ (s, 1H), $7.33(\mathrm{~s}, 1 \mathrm{H}), 6.89(\mathrm{~m}, 1 \mathrm{H}), 6.38(\mathrm{~s}, 1 \mathrm{H}), 6.31(\mathrm{~d}, J=15.7 \mathrm{~Hz}, 1 \mathrm{H}), 6.09(\mathrm{~d}, J=15.7 \mathrm{~Hz}, 1 \mathrm{H})$, $5.81(\mathrm{dd}, J=11.4,2.5 \mathrm{~Hz}, 1 \mathrm{H}), 5.74(\mathrm{dd}, J=15.7,7.3 \mathrm{~Hz}, 1 \mathrm{H}), 5.63(\mathrm{~d}, J=8.9 \mathrm{~Hz}, 1 \mathrm{H}), 5.47$ (ddd, $J=10.2,10.2,3.1 \mathrm{~Hz}, 1 \mathrm{H}), 5.38(\mathrm{~s}, 1 \mathrm{H}), 5.19(\mathrm{~s}, 1 \mathrm{H}), 4.90(\mathrm{dd}, J=8.5,4.6 \mathrm{~Hz}, 1 \mathrm{H}), 4.79(\mathrm{~s}$, 1H), $4.62(\mathrm{dd}, J=11.2,4.5 \mathrm{~Hz}, 1 \mathrm{H}), 4.34(\mathrm{br} \mathrm{s}, 1 \mathrm{H}), 4.28(\mathrm{~m}, 1 \mathrm{H}), 4.08(\mathrm{~m}, 1 \mathrm{H}), 3.95(\mathrm{~m}, 1 \mathrm{H})$, $3.82(\mathrm{~m}, 2 \mathrm{H}), 3.70(\mathrm{dd}, J=13.3,6.8 \mathrm{~Hz}, 1 \mathrm{H}), 3.51(\operatorname{app~t}, J=10.8 \mathrm{~Hz}, 1 \mathrm{H}), 3.45(\mathrm{~d}, J=10.0 \mathrm{~Hz}$, 1H), $3.37(\mathrm{~m}, 2 \mathrm{H}), 3.17(\mathrm{~d}, J=2.5 \mathrm{~Hz}, 1 \mathrm{H}), 3.16(\mathrm{~s}, 3 \mathrm{H}), 3.13(\mathrm{~s}, 3 \mathrm{H}), 2.83(\mathrm{~d}, J=12.6 \mathrm{~Hz}, 1 \mathrm{H})$, $2.67(\mathrm{~m}, 1 \mathrm{H}), 2.59(\mathrm{dd}, J=16.7,5.7 \mathrm{~Hz}, 1 \mathrm{H}), 2.49(\mathrm{~m}, 2 \mathrm{H}), 2.40(\mathrm{~m}, 4 \mathrm{H}), 2.15(\mathrm{~s}, 3 \mathrm{H}), 2.10(\mathrm{~m}$, 2H), $2.03(\mathrm{~m}, 3 \mathrm{H}), 1.95(\operatorname{app~t}, J=11.8 \mathrm{~Hz}, 1 \mathrm{H}), 1.90(\mathrm{~s}, 3 \mathrm{H}), 1.51(\mathrm{~m}, 4 \mathrm{H}), 1.15(\mathrm{~m}, 21 \mathrm{H}), 1.06$ (app t, $J=7.9 \mathrm{~Hz}, 9 \mathrm{H}), 1.04(\mathrm{~d}, J=6.9 \mathrm{~Hz}, 3 \mathrm{H}), 0.93(\mathrm{~m}, 1 \mathrm{H}), 0.79(\mathrm{~d}, J=6.4 \mathrm{~Hz}, 3 \mathrm{H}), 0.75$ (app $\mathrm{q}, J=8.1 \mathrm{~Hz}, 6 \mathrm{H}), 0.23(\mathrm{~s}, 9 \mathrm{H}) ;{ }^{13} \mathrm{CNMR}\left(125 \mathrm{MHz}, \mathrm{C}_{6} \mathrm{D}_{6}\right) \delta 165.4,161.4,159.8,144.9,142.5$, $140.9,138.7,137.7,137.3,136.6,134.7,134.3,133.0,121.1,119.4,119.1,110.2,99.8,97.5$, $89.6,86.5,80.7,80.0,78.5,74.4,74.1,73.4,73.3,71.3,68.9,66.8,56.4,55.1,42.4,41.8,41.4$, $39.8,37.5,34.5,32.8,31.8,30.7,30.2,30.1,27.5,22.9,18.4,18.3,14.2,13.7,13.4,12.8,7.3$, $7.0,6.7,6.1,2.3$; high resolution mass spectrum $\left(\mathrm{ES}^{+}\right) \mathrm{m} / \mathrm{z} 1293.7257\left[(\mathrm{M}+\mathrm{Na})^{+}\right.$; calcd for $\mathrm{C}_{70} \mathrm{H}_{110} \mathrm{~N}_{2} \mathrm{O}_{13} \mathrm{Si}_{3} \mathrm{Na:}$ 1293.7212]. 


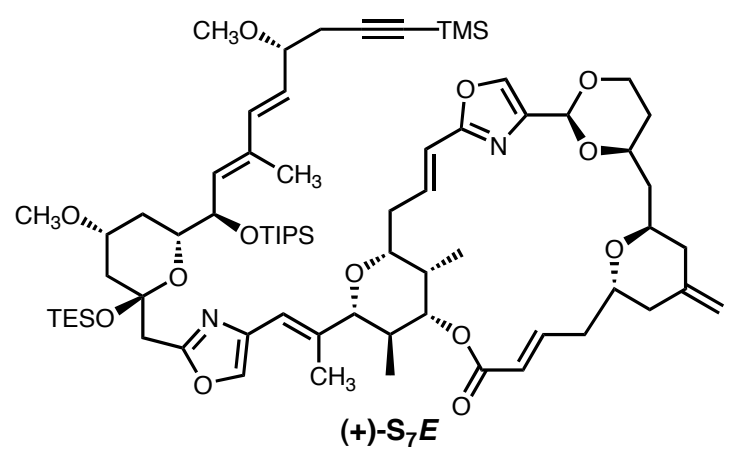

Protected E-C(2-3) Acetal Alkynyl Phorboxazole (+)-S ${ }_{7} E$ : E-C(2-3) macrocycle (+)-9E (4.6 mg, $0.007 \mathrm{mmol})$ was combined with C(33)-TES stannyl sidechain (-)-8 (9.2 mg, $0.010 \mathrm{mmol})$ in a flame-dried round-bottom flask $(5 \mathrm{~mL})$, azeotroped from benzene $(1 \mathrm{~mL}, 3 \mathrm{x})$ and dried under vacuum for two hours. Tris(dibenzylideneacetone)dipalladium-chloroform adduct $\left[\mathrm{Pd}_{2}(\mathrm{dba})_{3} \cdot \mathrm{CHCl}_{3}\right](1.4 \mathrm{mg}, 0.001 \mathrm{mmol})$, triphenylarsine $\left(\mathrm{AsPh}_{3}\right)(2.5 \mathrm{mg}, 0.008 \mathrm{mmol})$, and $\mathrm{Ph}_{2} \mathrm{PO}_{2} \mathrm{NBu}_{4}(4.7 \mathrm{mg}, 0.01 \mathrm{mmol})$ followed by DMF $(0.13 \mathrm{~mL}$, sparged with argon for thirty minutes) and $\mathrm{N}, \mathrm{N}$-diisopropylethylamine $(0.002 \mathrm{~mL}, 0.01 \mathrm{mmol})$ were added to the flask and the reaction stirred at room temperature under an argon atmosphere. After twenty hours, the light brown reaction mixture was introduced directly onto a silica gel column (40\% EtOAc/hexanes) to afford protected $E-\mathrm{C}(2-3)$ acetal alkynyl phorboxazole $(+)-S_{7} E(5.3 \mathrm{mg}, 60 \%$ yield $)$ as a light yellow oil. $[\alpha]_{D}^{20}+2.7\left(c\right.$ 0.1, $\left.\mathrm{CHCl}_{3}\right)$; IR (neat) 2929 (b), 2866 (b), 1717 (s), $1652(\mathrm{w}), 1464$ (s), 1247 (s), 1153 (s), 1099 (s), 1009 (b), 842 (s), 742 (s); ${ }^{1} \mathrm{HNMR}\left(500 \mathrm{MHz}, \mathrm{C}_{6} \mathrm{D}_{6}\right) \delta 7.33$ (s, 1H), $7.30(\mathrm{~s}, 1 \mathrm{H}), 6.70(\mathrm{~m}, 1 \mathrm{H}), 6.33(\mathrm{~s}, 1 \mathrm{H}), 6.30(\mathrm{~d}, J=15.7 \mathrm{~Hz}, 1 \mathrm{H}), 6.11(\mathrm{~d}, J=16.1 \mathrm{~Hz}, 1 \mathrm{H}), 6.05$ (d, $J=15.4 \mathrm{~Hz}, 1 \mathrm{H}), 5.73(\mathrm{dd}, J=15.7,7.4 \mathrm{~Hz}, 1 \mathrm{H}), 5.63(\mathrm{~d}, J=8.9 \mathrm{~Hz}, 1 \mathrm{H}), 5.36(\mathrm{~s}, 1 \mathrm{H}), 5.09$ (dd, $J=11.1,4.2 \mathrm{~Hz}, 1 \mathrm{H}), 4.89(\mathrm{dd}, J=8.6,4.7 \mathrm{~Hz}, 1 \mathrm{H}), 4.72(\mathrm{~s}, 1 \mathrm{H}), 4.66(\mathrm{~s}, 1 \mathrm{H}), 4.16(\mathrm{~m}, 2 \mathrm{H})$, $3.86(\mathrm{dd}, J=11.6,4.5 \mathrm{~Hz}, 1 \mathrm{H}), 3.81(\mathrm{~m}, 1 \mathrm{H}), 3.69(\mathrm{dd}, J=13.1,6.7 \mathrm{~Hz}, 1 \mathrm{H}), 3.43(\mathrm{~d}, J=9.9 \mathrm{~Hz}$, 1H), $3.37(\mathrm{~m}, 3 \mathrm{H}), 3.18(\mathrm{~m}, 1 \mathrm{H}), 3.15(\mathrm{~s}, 3 \mathrm{H}), 3.12(\mathrm{~s}, 3 \mathrm{H}), 2.58(\mathrm{dd}, J=16.7,5.7 \mathrm{~Hz}, 1 \mathrm{H}), 2.48$ (m, 2H), $2.39(\mathrm{~m}, 2 \mathrm{H}), 2.25(\mathrm{~m}, 3 \mathrm{H}), 2.16(\mathrm{~m}, 2 \mathrm{H}), 2.11(\mathrm{~s}, 3 \mathrm{H}), 1.92(\mathrm{dd}, J=12.9,3.1 \mathrm{~Hz}, 1 \mathrm{H})$, $1.89(\mathrm{~s}, 3 \mathrm{H}), 1.84($ app t, $J=12.3 \mathrm{~Hz}, 1 \mathrm{H}), 1.82(\mathrm{~m}, 1 \mathrm{H}), 1.72(\mathrm{~m}, 3 \mathrm{H}), 1.49(\mathrm{~m}, 3 \mathrm{H}), 1.33(\mathrm{~m}, 4 \mathrm{H})$, $1.17(\mathrm{~m}, 21 \mathrm{H}), 1.05(\operatorname{app~t}, J=7.9 \mathrm{~Hz}, 9 \mathrm{H}), 0.95(\mathrm{~d}, J=6.7 \mathrm{~Hz}, 3 \mathrm{H}), 0.86(\mathrm{~d}, J=6.5 \mathrm{~Hz}, 3 \mathrm{H}), 0.75$ (app q, $J=7.8 \mathrm{~Hz}, 6 \mathrm{H}), 0.23(\mathrm{~s}, 9 \mathrm{H}) ;{ }^{13} \mathrm{CNMR}\left(125 \mathrm{MHz}, \mathrm{C}_{6} \mathrm{D}_{6}\right) \quad \delta$ 183.0, 170.4, 166.7, 161.7, 
$159.7,146.6,142.1,140.9,138.7,137.8,137.3,136.5,135.7,135.4,134.7,132.9,131.8,126.7$, $123.7,118.9,118.8,110.9,104.4,99.8,95.5,89.6,86.5,80.7,78.9,77.1,74.4,74.1,73.3,71.4$, $70.3,66.4,56.4,55.1,42.4,41.8,40.9,40.4,38.5,34.7,34.6,31.8,30.2,27.5,18.4,18.3,14.2$, $13.7,13.4,12.8,7.4,6.7,0.3$; high resolution mass spectrum $\left(\mathrm{ES}^{+}\right) \mathrm{m} / \mathrm{z} 1293.7274\left[(\mathrm{M}+\mathrm{Na})^{+}\right.$; calcd for $\mathrm{C}_{70} \mathrm{H}_{110} \mathrm{~N}_{2} \mathrm{O}_{13} \mathrm{Si}_{3} \mathrm{Na:}$ 1293.7212].

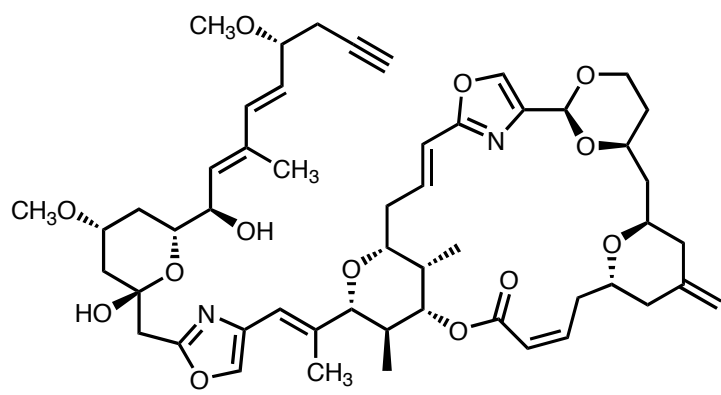

$(+)-7 Z$

Z-C(2-3) C(11-15)-Acetal Alkynyl Phorboxazole (+)-7Z: Protected Z-C(2-3) acetal alkynyl phorboxazole $(+)-\mathbf{S}_{7} \boldsymbol{Z}(6.7 \mathrm{mg}, 0.005 \mathrm{mmol})$ was dissolved in freshly distilled tetrahydrofuran (THF) $(1.3 \mathrm{~mL})$ under argon at $0{ }^{\circ} \mathrm{C}$. After stirring for five minutes tetrabutylammonium fluoride (TBAF) $(1.0 \mathrm{M} / \mathrm{THF}, 0.021 \mathrm{~mL}, 0.02 \mathrm{mmol})$ was added dropwise via syringe. After one hour, the reaction was quenched via dropwise addition of saturated aqueous sodium bicarbonate $(1.5 \mathrm{~mL})$ and warmed to room temperature. The layers of the biphasic mixture were separated and the aqueous layer was extracted with EtOAc $(3 \mathrm{~mL}, 4 \mathrm{x})$. The combined organic extracts were dried over $\mathrm{MgSO}_{4}$, filtered and concentrated under reduced pressure. Purification via silica gel chromatography (100\% EtOAc) afforded Z-C(2-3) C(11-15)acetal alkynyl phorboxazole $(+)-7 Z(3.7 \mathrm{mg}, 80 \%$ yield $)$ as an amorphous solid. $[\alpha]_{\mathrm{D}}{ }^{20}+37.4(\mathrm{C}$ 0.1, $\mathrm{CHCl}_{3}$ ); IR (neat) 3356 (b), 2940 (b), 2356 (w), 1712 (s), 1455 (s), 1365 (s), 1230 (s), 1175 (s), 903 (s), 848 (s), 735 (s); ${ }^{1} \mathrm{HNMR}\left(500 \mathrm{MHz}, \mathrm{C}_{6} \mathrm{D}_{6}\right) \delta 7.53(\mathrm{~s}, 1 \mathrm{H}), 7.03(\mathrm{~s}, 1 \mathrm{H}), 6.90(\mathrm{~m}, 1 \mathrm{H})$, $6.23(\mathrm{~d}, J=15.9 \mathrm{~Hz}, 1 \mathrm{H}), 6.21(\mathrm{~s}, 1 \mathrm{H}), 6.11(\mathrm{~d}, J=15.9 \mathrm{~Hz}, 1 \mathrm{H}), 5.82(\mathrm{dd}, J=11.5,2.2 \mathrm{~Hz}, 1 \mathrm{H})$, 5.59 (dd, $J=15.7,7.6 \mathrm{~Hz}, 1 \mathrm{H}), 5.56$ (d, $J=8.9 \mathrm{~Hz}, 1 \mathrm{H}$ ), 5.45 (ddd, $J=13.1,10.1,3.0 \mathrm{~Hz}, 1 \mathrm{H}$ ), $5.38(\mathrm{~d}, J=0.7 \mathrm{~Hz}, 1 \mathrm{H}), 5.20(\mathrm{~s}, 1 \mathrm{H}), 4.80(\mathrm{~s}, 1 \mathrm{H}), 4.62(\mathrm{dd}, J=11.2,4.3 \mathrm{~Hz}, 1 \mathrm{H}), 4.35(\mathrm{~m}, 2 \mathrm{H})$, 
$4.08(\mathrm{~m}, 1 \mathrm{H}), 3.96(\mathrm{~m}, 2 \mathrm{H}), 3.83(\mathrm{dd}, J=11.4,4.5 \mathrm{~Hz}, 1 \mathrm{H}), 3.78(\mathrm{~m}, 1 \mathrm{H}), 3.63(\mathrm{dd}, J=13.7,6.6$ Hz, 1H), $3.51($ app t, $J=11.2 \mathrm{~Hz}, 1 \mathrm{H}), 3.40(\operatorname{app~t}, J=9.9 \mathrm{~Hz}, 1 \mathrm{H}), 3.39(\mathrm{~m}, 1 \mathrm{H}), 3.09(\mathrm{~s}, 3 \mathrm{H})$, $3.07(\mathrm{~s}, 3 \mathrm{H}), 2.84(\mathrm{~d}, J=15.2 \mathrm{~Hz}, 1 \mathrm{H}), 2.69(\mathrm{~d}, J=15.1 \mathrm{~Hz}, 1 \mathrm{H}), 2.68(\mathrm{~m}, 1 \mathrm{H}), 2.46(\mathrm{~m}, 1 \mathrm{H}), 2.40$ (m, 5H), 2.31 (dd, $J=6.6,2.7 \mathrm{~Hz}, 1 \mathrm{H}), 2.28(\mathrm{dd}, J=11.2,3.0 \mathrm{~Hz}, 1 \mathrm{H}), 2.00(\mathrm{~m}, 4 \mathrm{H}), 1.95(\mathrm{~s}, 3 \mathrm{H})$, $1.78(\operatorname{app~t}, J=2.7 \mathrm{~Hz}, 1 \mathrm{H}), 1.65(\mathrm{~d}, J=0.9 \mathrm{~Hz}, 3 \mathrm{H}), 1.51(\mathrm{~m}, 3 \mathrm{H}), 1.31(\mathrm{~m}, 4 \mathrm{H}), 1.05(\mathrm{~d}, J=6.9$ $\mathrm{Hz}, 3 \mathrm{H}), 0.9(\mathrm{~m}, 2 \mathrm{H}), 0.7(\mathrm{~d}, J=6.4 \mathrm{~Hz}, 3 \mathrm{H}) ;{ }^{13} \mathrm{CNMR}\left(125 \mathrm{MHz}, \mathrm{C}_{6} \mathrm{D}_{6}\right) \delta 161.0,145.3,142.7$, $141.1,138.9,137.7,137.1,136.1,134.9,134.3,131.8,129.8,121.2,119.7,118.5,110.1,97.7$ $97.1,89.5,80.9,80.1,78.7,73.6,73.5,73.1,71.2,70.5,69.1,67.0,56.5,55.5,41.6,41.3,41.2$, $40.0,37.7,35.8,34.7,33.7,33.0,32.3,32.2,30.9,30.4,30.3,30.1,29.9,26.4,25.8,14.5,13.5$, 6.3; high resolution mass spectrum $\left(\mathrm{ES}^{+}\right) \mathrm{m} / \mathrm{z} 951.4637\left[(\mathrm{M}+\mathrm{Na})^{+}\right.$; calcd for $\mathrm{C}_{52} \mathrm{H}_{68} \mathrm{~N}_{2} \mathrm{O}_{13} \mathrm{Na}$ : 951.4618].

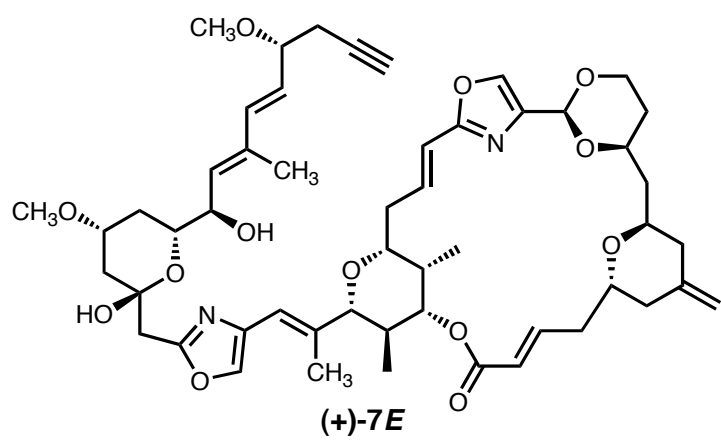

E-C(2-3) C(11-15)-Acetal Alkynyl Phorboxazole (+)-7E: Protected E-C(2-3) acetal alkynyl phorboxazole $(+)-S_{7} E(0.010 \mathrm{~g}, 0.008 \mathrm{mmol})$ was dissolved in freshly distilled tetrahydrofuran (THF) $(1.9 \mathrm{~mL})$ under argon at $0{ }^{\circ} \mathrm{C}$. After stirring for five minutes, tetrabutylammonium fluoride (TBAF) $(1.0 \mathrm{M} / \mathrm{THF}, 0.031 \mathrm{~mL}, 0.03 \mathrm{mmol})$ was added dropwise via syringe. After one hour, the reaction was quenched via dropwise addition of saturated aqueous sodium bicarbonate $(2.0 \mathrm{~mL})$ and allowed to warm to room temperature. The layers of the biphasic mixture were separated and the aqueous layer was extracted with EtOAc $(3 \mathrm{~mL}, 4 \mathrm{x})$, dried over $\mathrm{MgSO}_{4}$, filtered and concentrated under reduced pressure. Purification via silica gel chromatography (100\% EtOAc) afforded E-C(2-3) C(11-15)-acetal alkynyl phorboxazole (+)-7E 
(6.0 $\mathrm{mg}, 81 \%$ yield) as an amorphous solid. $[\alpha]_{\mathrm{D}}{ }^{20}+21.8\left(c 0.1, \mathrm{CH}_{2} \mathrm{Cl}_{2}\right)$, IR (neat) 3305 (b), 2926 (b), 2853 (s), 1714 (s), 1655 (s), 1456 (b), 1362 (b), 1153 (s), 1097 (s), 1010 (b), 879 (w); 'HNMR $\left(500 \mathrm{MHz}, \mathrm{C}_{6} \mathrm{D}_{6}\right) \delta 7.40(\mathrm{~s}, 1 \mathrm{H}), 7.31(\mathrm{~s}, 1 \mathrm{H}), 6.83(\mathrm{~m}, 1 \mathrm{H}), 6.31(\mathrm{~d}, J=15.8 \mathrm{~Hz}, 1 \mathrm{H}), 6.26(\mathrm{~s}, 1 \mathrm{H})$, $6.22(\mathrm{~d}, J=16.1 \mathrm{~Hz}, 1 \mathrm{H}), 6.16(\mathrm{~d}, J=15.4 \mathrm{~Hz}, 1 \mathrm{H}), 5.67(\mathrm{dd}, J=15.6,7.5 \mathrm{~Hz}, 1 \mathrm{H}), 5.64(\mathrm{~d}, J=$ $8.8 \mathrm{~Hz}, 1 \mathrm{H}), 5.52(\mathrm{~s}, 1 \mathrm{H}), 5.45(\mathrm{~s}, 1 \mathrm{H}), 5.19(\mathrm{dd}, J=11.1,4.1 \mathrm{~Hz}, 1 \mathrm{H}), 4.82(\mathrm{~s}, 1 \mathrm{H}), 4.75(\mathrm{~s}, 1 \mathrm{H})$, $4.43($ app t, $J=7.3 \mathrm{~Hz}, 1 \mathrm{H}), 4.29(\mathrm{~m}, 1 \mathrm{H}), 4.05(\mathrm{dd}, J=10.2,4.5 \mathrm{~Hz}, 1 \mathrm{H}), 3.95$ (dd, $J=11.7,4.5$ $\mathrm{Hz}, 1 \mathrm{H}), 3.87(\mathrm{~m}, 1 \mathrm{H}), 3.72(\mathrm{dd}, J=14.1,7.3 \mathrm{~Hz}, 1 \mathrm{H}), 3.47(\mathrm{~m}, 3 \mathrm{H}), 3.28(\operatorname{app} \mathrm{t}, J=10.2 \mathrm{~Hz}, 1 \mathrm{H})$, $3.18(\mathrm{~s}, 3 \mathrm{H}), 3.16(\mathrm{~s}, 3 \mathrm{H}), 2.92(\mathrm{~d}, J=15.4 \mathrm{~Hz}, 1 \mathrm{H}), 2.78(\mathrm{~d}, J=15.4 \mathrm{~Hz}, 1 \mathrm{H}), 2.55$ (dd, $J=5.8$, $2.7 \mathrm{~Hz}, 1 \mathrm{H}), 2.52(\mathrm{dd}, J=5.8,2.6 \mathrm{~Hz}, 1 \mathrm{H}), 2.50(\mathrm{~m}, 1 \mathrm{H}), 2.44(\mathrm{dd}, J=6.6,2.7 \mathrm{~Hz}, 1 \mathrm{H}), 2.40$ (dd, $J=6.7,2.8 \mathrm{~Hz}, 1 \mathrm{H}), 2.34(\mathrm{~m}, 4 \mathrm{H}), 2.18(\mathrm{~m}, 2 \mathrm{H}), 2.11(\mathrm{~m}, 1 \mathrm{H}), 2.04(\mathrm{~m}, 2 \mathrm{H}), 1.99(\mathrm{~s}, 3 \mathrm{H}), 1.92(\mathrm{~m}$, 1H), $1.87($ app t, $J=2.6 \mathrm{~Hz}, 1 \mathrm{H}), 1.81(\mathrm{~m}, 3 \mathrm{H}), 1.74(\mathrm{~s}, 3 \mathrm{H}), 1.61(\mathrm{~m}, 2 \mathrm{H}), 1.36(\mathrm{~m}, 3 \mathrm{H}), 1.07(\mathrm{~d}, J$ $=6.7 \mathrm{~Hz}, 3 \mathrm{H}), 1.01(\mathrm{~m}, 2 \mathrm{H}), 0.92(\mathrm{~d}, J=6.5 \mathrm{~Hz}, 1 \mathrm{H}) ;{ }^{13} \mathrm{CNMR}\left(125 \mathrm{MHz}, \mathrm{C}_{6} \mathrm{D}_{6}\right) \delta 167.3,162.4$, $161.3,147.3,142.6,141.5,139.4,138.7,138.1,137.4,136.4,136.3,135.9,132.2,124.3,119.5$ $118.8,111.5,97.4,96.1,89.9,81.5,81.2,79.4,77.8,73.9,73.4,71.6,70.9,66.9,56.8,55.9$, $41.7,41.4,41.1,40.4,39.0,38.6,35.2,35.1,34.1,33.1,32.3,30.7,30.6,30.6,26.8,23.6,14.9$, 14.0, 13.9, 6.7; high resolution mass spectrum $\left(\mathrm{ES}^{+}\right) \mathrm{m} / \mathrm{z} 951.4636\left[(\mathrm{M}+\mathrm{Na})^{+}\right.$; calcd for $\mathrm{C}_{52} \mathrm{H}_{68} \mathrm{~N}_{2} \mathrm{O}_{13} \mathrm{Na:}$ 951.4669]. 